JOURNAL OF MATERIALS SCIENCE, VOL 45, 2010, 3882-3894

\title{
PARTICLE CAVITATION IN RUBBER TOUGHENED EPOXIES: THE ROLE OF PARTICLE SIZE
}

\author{
F.J. Guild*, A.J. Kinloch and A.C. Taylor, \\ Department of Mechanical Engineering, \\ Imperial College London, South Kensington Campus, London SW7 2AZ, UK.
}

\begin{abstract}
Rubber toughened epoxies are used in a wide range of applications including adhesives when toughness is a crucial property. It is well known that the cavitation of the rubber particles is an important process to optimise the toughness of such materials. This paper describes the development of a predictive model to describe the dependence of rubber particle cavitation on particle size. The model is developed using a combination of experimental observations and finite element simulations. Predictions have been obtained for both uniaxial loading conditions and the triaxial loading conditions expected ahead of a crack. The model has been extended to consider the cavitation of nano-sized 'rubber' particles.
\end{abstract}

Keywords: rubber toughened epoxies, toughness, particle cavitation

\section{Introduction}

Current developments in rubber toughened epoxies include reinforcement using nano-scale particles which can be used alone or with other more conventionally sized particles. These materials are being used in a wide range of applications including adhesives and it is well known that toughness is a crucial property for successful adhesive joint design [e.g. 1]. The work presented in this paper is part of a wider project investigating the mechanical behaviour and toughness of these materials through both experimental investigations and numerical modelling.

Cavitation of the rubber particle is clearly observed experimentally for conventionally (i.e. micro-) scaled materials and it is generally accepted that this process is crucial for a very significant 
enhancement of the toughness [e.g.1-7]. The process of particle cavitation leads to changes in the stress state in the surrounding epoxy matrix which gives rise to increased plastic deformation and plastic hole growth. These processes are clearly found in our finite element simulations of the conditions ahead of a crack. The energy associated with these processes has indeed been shown to be associated with the enhancement of toughness. However, the occurrence of rubber particle cavitation has been less well defined for nano-scaled particles, and the ability of the rubber particle to undergo cavitation may be related to its size.

The development of a criterion for the cavitation of rubber particles is a crucial step in the final goal of this work, namely the development of a methodology for the prediction of toughness. The different criteria have been reviewed by Fond [3]. The importance of understanding the processes affecting cavitation has been highlighted in a recent review of rubber-toughened epoxies [8]. It is notable that many models described in the literature for particle cavitation are related to the volume strain energy stored in the particle which is believed to drive the process of cavitation. This volume strain energy is directly related to the hydrostatic stress in the particle. The major source of energy dissipation may be considered to be dominated by the surface tension [2] or the fracture energy for the breakage of rubber molecular chains [4]. For uniaxial tension and assuming no interaction between the particles, finite element simulations were developed which explored the role of plasticity of the matrix, and demonstrated that, for large rubber particles, cavitation is expected to occur at a hydrostatic stress level within the rubber particles of around $20 \mathrm{MPa}$ [9]. More recently, cavitation for nano-sized rubber particles has been observed and analysed using an energy based approach [10].

The results in this paper have been derived using finite element simulations based on experimental observations. The results from the finite element simulations have been used to deduce a criterion for particle cavitation, using an energy based approach, both for uniaxial tension and for the triaxial stress conditions ahead of a crack. The results lead to prediction of a particle size effect for cavitation both for uniaxial loading and for the triaxial loading conditions ahead of a crack. The toughness of rubber toughened epoxies may be directly related to the size of the rubber particles. The model has been extended to include preliminary results for the cavitation of nano-sized 'rubber' particles.

\section{Finite Element Model}

\subsection{Microstructural Model and Boundary Conditions}


The finite element model is based on the unit cell approach. The present models assume uniform distribution of the particles, which are all at equal inter-particle distance. This assumption is the most logical arrangement for a uniform distribution. This distribution is achieved from the face-centred cubic particle arrangement. The face-centred cubic unit cell has 1/8 symmetry as shown in Figure 1a; simulations were carried out for the unit cell shown in Figure 1b. Typical simulations used around 31000 solid, tetrahedral, first order elements.

Boundary conditions can be deduced from the symmetry assumptions for the array of unit cells. All faces of the cell must always remain straight and parallel to their original plane. Three orthogonal faces remain fixed. The loading on the opposite faces for triaxial loading is shown in Figure 1b. For uniaxial loading, the top surface (Figure 1b) only is displaced and Poisson's contractions are allowed for the other two faces, with constraint equations used to maintain the faces straight and parallel to their original plane.

\subsection{Material Properties}

The material modelled here is a 'model' rubber toughened epoxy prepared in the laboratory. The particle size and content have been measured; the mean particle radius is $1.7 \mu \mathrm{m}$ and the overall rubber content is $20 \%$ by volume. The material properties for the constituents have been carefully considered. The properties of the rubber were matched to experimental results from the literature [11] using a two parameter hyperelastic model with initial bulk modulus set to $3 \mathrm{GPa}$. Hybrid formulation elements, which include an extra degree of freedom for hydrostatic displacement, are required for this material model. Figure 2a compares the experimental stress/strain results with the predicted results for this material model; close agreement is found over a very wide range of strain.

The epoxy matrix properties are based on the exponent Drucker-Prager model available in ABAQUS Standard [12]. The exponent model was required to allow convergence at high values of applied strain. The model was derived using experimentally measured tensile and shear stress/strain behaviour. Figure 2b compares the tensile experimental stress/strain measurements with the predicted results for this material model; reasonable agreement is found.

\subsection{Loading}

The simulations have been carried out for both uniaxial loading and for triaxial loading, including the conditions expected ahead of a crack. These triaxial conditions are defined as a loading ratio of 
1:0.8:0.8 in the $\mathrm{y}, \mathrm{x}$ and $\mathrm{z}$ directions respectively (see Figure 1b). The cavitation of rubber particles was simulated by removal of elements; the process is assumed to take place at constant applied strain. For uniaxial loading, the strain in the loading direction was maintained while the orthogonal directions changed displacement since the overall Poisson's ratio is changed by the removal of the rubber (see Section 5.1). For triaxial loading, the maximum strain was maintained to reflect the applied strain, and the strains in the orthogonal directions were changed to maintain the loading ratio.

\section{Thermal Strains}

\subsection{Development of Thermal Strains}

Thermal strain is expected to exist within particulate filled composites if the preparation of the material involves high temperatures. On cooling, thermal stresses arise from the difference in the values of coefficients of thermal expansion for the particles and matrix. At ambient temperature, the rubber particles have a higher value of the coefficient of thermal expansion than the glassy epoxy matrix; thermal contraction would thus place the rubber particles in tension.

The values of coefficient of thermal expansion for the epoxy and rubber were gained from the literature: $60 \times 10^{-6} /{ }^{\circ} \mathrm{C}$ for the epoxy [13]; $16 \times 10^{-5} /{ }^{\circ} \mathrm{C}$ for the rubber [14]. These values are quoted as values at ambient temperature; these values were used throughout the temperature range in these simulations.

\subsection{Preparation of Adhesive Specimens}

The adhesive is prepared as described by Oba [15]. Specimens are cured at $160^{\circ} \mathrm{C}$ in a steel mould and then left to cool slowly in the oven. The process of cooling has been monitored using thermocouples. As the temperature decreases, the adhesive passes through its glass transition temperature as shown in Figure 3a. The shear modulus of the adhesive through its glass transition temperature has been measured using Dynamic Mechanical Thermal Analysis [15]; the data are shown in Figure 3b. The point of inflection of the modulus versus temperature data has been determined; $80.5^{\circ} \mathrm{C}$. Above this temperature, it is assumed that the thermal strains are able to relax. Thus the thermal residual stress as the temperature falls from $80.5^{\circ} \mathrm{C}$ to ambient temperature, $20^{\circ} \mathrm{C}$ must be found. We have assumed that the variability in Young's modulus is identical to the measured variability in shear modulus.

\subsection{Simulations to determine Thermal Residual Stress}


The temperature change and modulus change must be input to the analysis on the same time scale. Figures 4a shows the measured cooling rate between $80.5^{\circ} \mathrm{C}$ and $20^{\circ} \mathrm{C}$ extracted from Figure 3a and plotted against normalised time. Using the relationship between temperature and time in Figure 4a, modulus results between the temperature limits were extracted from Figure $3 \mathrm{~b}$ and plotted as a function of the same normalised time scale; the values of modulus have been normalised with respect to the modulus at $20^{\circ} \mathrm{C}$. The results are shown in Figure $4 \mathrm{~b}$. It is clear that the fastest rates of cooling and stiffness change occur over the same short time range.

The data in Figure 4b indicate that the modulus variability must be taken into account in order to predict the thermal stress. The most elegant way to model time dependent stiffness variability is to use a viscoelastic analysis. However, the variability in stiffness must then be input as a Prony series, expressed as a sum of negative exponentials, and the form of the variability shown in Figure 4b cannot be fitted to any sum of such functions. The variability in modulus was therefore reduced to three portions of linear variability, shown by the red dashed lines in Figure 4b. The fit is reasonable particularly at the start of the time range when most temperature change occurs.

Three separate elastic analyses were carried out using the average modulus for each portion of linear variability and the corresponding temperature drop for that modulus change derived from the data in Figure 4b. The total thermal stress was calculated as the sum of the stresses from the three analyses. The von Mises stress contours arising from the total thermal stress are shown in Figure 5. The stress in the epoxy is fairly high at the interface, i.e. 16.7 MPa, but falls sharply away from the interface; the strain energy associated with this stress state is small. The rubber particles are in significant uniform hydrostatic tension of 9.17 MPa. The total hydrostatic thermal strain of the unit cell is 0.0089 .

\section{Experimental Results}

Tensile tests have been carried out to observe the strain for the onset of whitening; this phenomenon has been linked with the onset of particle cavitation [16]. Quasi-static tensile tests were carried out using specimens of the rubber toughened epoxy. The tests were conducted at room temperature and at a test rate of $1 \mathrm{~mm} / \mathrm{min}$. The colour of the specimen was observed using a video camera, and the occurrence of whitening was clearly observed in subsequent examination of the film. Clear whitening was observed when the value of applied tensile strain was 0.024 . This value is supported by the onset of particle cavitation for this material under tensile loading for this material having been observed at this strain from measurements of Poisson's ratio [17]. These experimental results have been compared 
with our predictions (see Section 5.1).

\section{Finite Element Results}

\subsection{Comparison with Experimental Results}

The tensile test has been simulated with particle cavitation taking place at applied strain of 0.024 and then continued loading; the predicted stress/strain behaviour is shown in Figure 6a and the predicted values of Poisson's ratio are compared with experimental measurements from the literature [17] in Figure 6b. Very good agreement is found, and the reduction in values of Poisson's ratio associated with rubber particle cavitation is seen which causes the effect on the boundary conditions, as described in Section 2.3. As expected, the cavitation taking place at constant strain causes a reduction in the load, reflecting the changing stored energy. These independent experimental results show that particle cavitation occurs at a strain of 0.024 , as found in the tensile tests carried out for this work.

\subsection{Plastic Hole Growth}

The cavitation of the rubber particles causes the void to grow. This arises since the stress state within the particles prior to cavitation is almost pure hydrostatic tensile stress, for the application of both uniaxial and triaxial stress. The rubber material is very stiff in hydrostatic tension, so the process of cavitation leads to a reduction in constraint and growth of the subsequent void; the results are shown in Figure 7. The results are presented as the 'Relative Volume' of the particle or void: this is the volume normalised with respect to the initial volume of the rubber particle at phase separation, before thermal contraction. These initial thermal contractions cause reduction in volume of the rubber particle. The process of rubber particle cavitation has been simulated to occur at different values of strain; the results in Figure 7 show the volume of the rubber particle just prior to cavitation and the volume of the subsequent void following cavitation at a range of strains. The process of cavitation has been modelled by the removal of the elements representing the particle and the modification of the boundary conditions to reflect the changed value of Poisson's ratio (see Figure 6b). The volumes of both the particle and subsequent void increase with increasing strain for both uniaxial and triaxial loading, although the increase is far more pronounced for triaxial loading. There is only a small increase in particle or void volume for uniaxial loading, but the shape of the void is an ellipsoid. For triaxial loading the shape of the void is more spherical. The volume change during cavitation under triaxial loading at high strains is around $10 \%$. However, these volume changes are smaller than the volume change observed on fracture surfaces $[6,18]$ since, as observed in our finite element simulations, much further void growth occurs after cavitation arising from the plastic deformation of the matrix. 


\subsection{Growth of Shear Bands}

The cavitation of the rubber particles is expected to cause relief of constraint and a reduction in the triaxiality of the stress state within the surrounding matrix. Figures 8 and 9 show the contours of von Mises stress within the matrix before and after cavitation under uniaxial and triaxial loading. The applied strains are those corresponding to the cavitation of particles in this material with initial rubber sphere radius of $1.7 \mu \mathrm{m}$, i.e. 0.024 for uniaxial loading and 0.0116 for triaxial loading (see section 6.3). Unexpectedly, the results in Figure 8 for uniaxial loading show that no growth of shear bands is promoted by the rubber particle cavitation. Before the cavitation there is a shear band forming between the particles (see figure 8a), but there is a reduction in its intensity and a reduction in the value of maximum von Mises stress between the particles after cavitation (see figure $8 \mathrm{~b}$ ). It is notable that the values of von Mises stress at the particle interface are very similar before and after cavitation; it is the distribution of von Mises stress between the particles which is affected by the process of cavitation. Further results at higher strains have been examined, and this effect of reduction in shear banding between the particles was found for cavitation at all strain levels. In contrast, the results in Figure 9 clearly show the higher values of maximum von Mises stress and the growth of shear bands between the particles after the cavitation of the rubber particles under triaxial loading. Rubber particle cavitation has been shown to promote the growth of shear bands in triaxial loading. This same effect of shear band promotion after particle cavitation was found for results from cavitation at higher strains.

\subsection{Stress in Rubber Particles before Cavitation}

Observation of the stress state within the particles for both stress conditions shows that the particles are in almost pure uniform hydrostatic tensile stress. This stress state is shown in Figures 8a and 9a: the value of von Mises stress within the particles is near zero. A similar stress state within rubber particles has been observed for unidirectional loading in other unit cells [19]. However, the magnitude of the stress is far higher for the application of triaxial stress; the values are compared in Figure 10. For the application of triaxial stress, the results are plotted as a function of the applied maximum strain since this can be related to the globally applied strain. It is notable that very high values of hydrostatic stress are predicted for the application of triaxial stress.

Consideration of the results in Figure 10 indicate that a consistent criterion for rubber particle cavitation in uniaxial tension and ahead of a crack is not expected to be associated with the value of 
hydrostatic tensile stress in the particle. Cavitation in the uniaxial tests is observed at 0.024 applied strain; the results in Figure 10 show that the hydrostatic tensile stress in the rubber particles at this strain level is 22.3 MPa. The results in Figure 6 show that using triaxial loading, this stress level in the rubber particles is reached when the maximum applied strain is 0.0029. This result is independent of the size of the particle. Particle cavitation ahead of a crack for such a low level of applied strain does not appear credible. This result implies that cavitation would occur around any small crack or defect at the very onset of application of load. This is not generally observed experimentally for either conventional-scale or nano-scale reinforcements. Hence a more realistic criterion for rubber particle cavitation is required.

\section{Cavitation Criterion}

\subsection{Development of Cavitation Criterion}

An alternative criterion for rubber particle cavitation has been sought based on an energy balance approach. The experimental observations of rubber particles of $1.7 \mu \mathrm{m}$ radius undergoing cavitation at 0.024 applied uniaxial strain has been simulated and used to determine the unknown value of surface energy of the void. The value deduced is comparable with literature values of fracture energy of rubber, as described in Section 7. This value of surface energy has then been used to analyse further simulations in both uniaxial and triaxial loading to allow deduction of a particle size dependent criterion for cavitation of rubber particles.

\subsection{Simulation of Cavitation in Uniaxial Tension}

The process of cavitation in the uniaxial test followed by unloading was simulated; the results are shown in Figure 11. Particle cavitation is simulated at 0.024 applied strain, as observed experimentally. The process of cavitation was simulated within the finite element analyses using removal of the elements representing the rubber particles. The applied strain was maintained during this step, and the load carried was therefore reduced. Following the cavitation step, the load was incrementally removed; at zero load, residual strain is found indicating that plastic deformation has taken place.

The results in Figure 11 show that energy loss is associated with the cavitation process. The hypothesis of our model is that this energy contributes to the energy required for the cavitation process. This energy is represented by the area between the loading and unloading curves in Figure 11. The curves in Figure 11 were integrated, and the area between the curves was found. Alternatively this 
total energy loss can be calculated from the difference in available energy in the system before and after cavitation. This can be found from the finite element results using two alternative methods. The first method uses the same concepts as the integration of the curves: the energy before cavitation, that is the area below the loading curve, is the sum of the plastic and elastic energy; the energy after cavitation, that is the area under the unloading curve, is the recoverable (elastic) strain energy. Since the stiffness of the system is changed by the cavitation process, the elastic strain energy in the system changes as a result of the particle cavitation. The second method uses the value of external work left in the model after unloading. The values of total energy were calculated via numerical integration and using the two energy methods; all three values were found to be in agreement. The energy contributions are presented in Section 6.3 below.

\subsection{Cavitation Criterion}

The energy loss associated with the cavitation of a single particle can be found from Figure 11. The results for the finite element model are from a mesh size of $1 \mathrm{~mm}^{3}$ with particle radius of $0.457 \mathrm{~mm}$ for $20 \%$ volume fraction of particles; the unit cell contains half a particle. Scaling has been used to translate the results from the simulation to correspond to a whole particle of radius $1.7 \mu \mathrm{m}$, corresponding to the material tested. This energy is described as the total energy, $\mathrm{U}_{\mathrm{T}}$. The components of energy used in the processes of cavitation are:

- The change in elastic energy in the matrix, $\Delta \mathrm{U}_{\mathrm{e}}$

- $\quad$ The change in plastic energy in the matrix, $\Delta \mathrm{U}_{\mathrm{p}}$

- $\quad$ The surface energy to form the resulting void, $\mathrm{U}_{\mathrm{s}}$

An additional component of energy must be available to the process, namely the release of energy from the rubber particle on cavitation, $U_{\text {rub }}$. Thus the energy balance for the process of cavitation is:

$$
\mathrm{U}_{\mathrm{T}}=\Delta \mathrm{U}_{\mathrm{e}}+\Delta \mathrm{U}_{\mathrm{p}}+\mathrm{U}_{\mathrm{s}}-\mathrm{U}_{\mathrm{rub}}
$$

This cavitation criterion was applied to the results shown in Figure 11 and the equation was solved to find the surface energy value of the void: $0.649 \mathrm{~J} \mathrm{~m}^{-2}$. This is a reasonable value assuming that the void is coated with rubber and taking into account the breaking of bonds required to allow the process of cavitation as discussed in Section 7.3 below.

\subsection{Energy Contributions}


The finite element results are used to calculate the components of energy dissipation associated with the cavitation of one rubber particle for the material studied here, with radius $1.7 \mu \mathrm{m}$. The different contributions for uniaxial and triaxial loading are shown in Figure 12a and b respectively; the uniaxial results are plotted as a function of uniaxial applied strain and the triaxial results are plotted as a function of maximum applied strain to the unit cell. For uniaxial loading, cavitation for particle radius of $1.7 \mu \mathrm{m}$ takes place at 0.024 applied strain; for triaxial loading it takes place at 0.0116 maximum applied strain. Visual inspection of the graphs confirms that solution of equation 1 occurs at these points. The relative significance of the energy contributions is discussed in Section 7.5 below.

\subsection{Particle Size Effect}

All the energy values in equation 1 are dependent on the applied strain at which rubber particle cavitation takes place. The values of total energy, $U_{T}$, the changes in elastic and plastic energy, $\Delta U_{e}$ and $\Delta U_{p}$, and the energy contributed from the rubber particle, $U_{\text {rub }}$, are all dependent on the volume of material, thus the cube of the particle radius. However, the surface energy to form the resulting void, $U_{s}$, is dependent on the area of the resulting void, thus, indirectly, dependent on the square of the particle radius. For all simulations, the value of $U_{s}$ was calculated using the correct size and shape of the void after cavitation. Thus solution of equation 1 for cavitation at a given strain yields the radius of the particle which would cavitate at that strain. The cavitation criterion expressed in equation 1 yields particle size dependence for the process of rubber particle cavitation.

Further finite element simulations under both uniaxial and triaxial loading have been carried out to determine the globally applied strain required to cause rubber particle cavitation for different particle sizes. The results are shown in Figure 13. As expected, lower applied strain is required to cause larger particles to cavitate. The results for the triaxial loading are plotted with respect to the maximum applied strain (not the total hydrostatic strain). Using this comparison, particles of a given size ahead of a crack cavitate at lower applied strain than those under uniaxial strain without a crack present.

\section{Discussion}

\subsection{Global Applied Strain}

The results of our cavitation model have been presented as functions of the strain applied to the unit cell analysed. For uniaxial loading, the strain applied to the unit cell is equal to the global applied 
strain to the material. The cavitation criterion for uniaxial loading is presented as a function of global applied uniaxial strain.

For triaxial loading the relationship is more complex. The three-dimensional stress state ahead of a crack has been examined from finite element simulations. These simulations are presented in detail in the derivation of our toughness model [20]. We observe that the value of applied stress around the plastic zone is typically around double the global applied stress remote from the crack. Around the crack, values of stress in the orthogonal directions are lower than the value of stress in the applied direction. Similar results have been found in previous finite element simulations [21] which were successfully compared with classical calculations [22]. Our triaxial loading conditions in the ratio 1:0.8:0.8 is an 'average' representation of the triaxial loading conditions around the crack. The value of maximum applied strain to the unit cell may be related to the global applied strain via a factor of two.

\subsection{Microstructural Model}

The finite element model has been deduced using the unit cell approach. This approach requires the assumption of a regular uniform distribution of particles. Observation of micrographs of such materials clearly show that such a distribution is not generally found. In previous work [19] a random distribution of particles was assumed. Statistical modelling of the random distribution, taking into account the actual particle size [23], allows the effect of varying inter-particle distances to be taken into account, but these effects are not significant for the low volume fraction of particles considered here. The fundamental assumption of this model was that the effects of neighbouring particles on the stress distributions around the analysed particle are smeared; directionality is not included. Thus this random model allows good predictions of global properties, but cannot be used to identify fracture behaviour when directionality is important. We have therefore developed this unit cell approach in order to include this directionality. The distribution we have chosen, namely the face-centred cubic arrangement, is the most logical choice since all particles are equidistant with respect to each other. We note that this is a simplified model for the microstructure, but the results may be considered as an 'average' of the predictions that would be obtained if the real, variable, inter-particle distances were considered. Thus, for a large number of particles, as would be expected to be within the cavitated zone ahead of a crack, the predictions made using this simplified model of the microstructure are valid.

\subsection{Cavitation Criterion}


Our prediction for particle cavitation is based on an energy balance; the energies have been deduced from the finite element simulations. Similar energy balance approaches have been used in several previous models [e.g. 2] and more recently applied to cavitation of nano-sized particles [10]. The value of surface energy leading to the formation of the void containing the cavitated rubber particle was deduced from the experimental observation that cavitation occurred at 0.024 strain. The value of surface energy deduced is $0.649 \mathrm{Jm}^{-2}$. This value is approximately 20 times higher than the surface energy measured for an existing rubber surface and used in previous analyses [24]. We consider that this difference arises since the surface energy value for this cavitation process should include the energy required to fracture the carbon-carbon bonds which allows the cavitation process to take place.

The energy required to form the rubber surface may be estimated as the energy required to tear the rubber. The tearing strength of various rubbers calculated from the energy to fracture the bonds has been found to be around $0.5 \mathrm{~J} \mathrm{~m}^{-2}\left(5 \times 10^{4} \mathrm{erg} \mathrm{cm}^{-2}\right)$ for a wide range of rubbers [25]. It is noted that this calculation ignores the energy arising from the deformation of the rubber. Thus we believe that our value of $0.649 \mathrm{Jm}^{-2}$ for the surface energy of the rubber, taking into account the deformation and fracture required to make the surface, is a credible value.

\subsection{Particle Size Dependence}

The dependence of particle size shown in Figure 13 appears credible for conventionally scaled materials. For the material tested here, with particle radius of $1.7 \mu \mathrm{m}$, cavitation in uniaxial tests is observed at 0.024 applied strain; this value of strain was measured in this work, and confirmed by independent measurements. In triaxial loading, rubber particle cavitation is predicted to occur at 0.0116 maximum applied strain. In the presence of a crack, this maximum applied strain may be associated with global applied strain of 0.0058 (see Section 7.1). Thus, ahead of a crack, whitening in rubber toughened adhesives with conventional sized particles is predicted at an applied strain of about 0.006; this is close to many experimental observations as discussed below.

The dependence of particle cavitation on particle size has been considered in the literature for many years. Early work includes description of a lack of toughness in a rubber toughened adhesive arising from a reduction in particle size [e.g. 26, 27]. The various quantitative results relating particle cavitation to particle size have been reviewed by Dompas et al [4]. Figure 14 compares the predictions made here for cavitation in uniaxial loading with those quantitative results; our predictions are generally in good agreement both with discrete values and with the overall trend. 
Several results from experimental investigations found in the literature suggest that optimised toughness is not found when the particle radius falls below around $200 \mathrm{~nm}$ [e.g. 7, 28, 29]. These observations are in general agreement with our predictions for particle cavitation under triaxial loading shown in Figure 13. However, as discussed by Liu et al [10], cavitation has been observed for nanosized 'rubber' particles with diameter of around $15 \mathrm{~nm}$. The analysis of Lazzeri and Bucknall [2] was re-worked to include the energy contribution from pre-existing nano-voids and was found to predict cavitation for such particles, including an extra energy contribution from pre-existing voids. It is notable that the value of surface energy for the rubber surface in the void used in the Lazzeri and Bucknall model [2] is $0.03 \mathrm{Jm}^{-2}$. As described in section 7.3 above, we consider that the value used for conventional rubber particles should be higher as it must include the energy required to break the carbon-carbon bonds. However, for these nano-sized particles, the 'rubber' is a self-assembled block copolymer [10]. Void growth or cavitation in such material could occur without failure or stretching of carbon-carbon bonds. We have repeated our analyses, for both uniaxial and triaxial loading, using a value of surface energy for the void of $0.03 \mathrm{Jm}^{-2}$; the results are shown in Figure 15 . These results show that cavitation of nano-sized 'rubber' particles which cavitate without the requirement to stretch or break carbon-carbon bonds is predicted using our approach which includes all the energy contributions arising from the void process. For the 'rubber' particles of $15 \mathrm{~nm}$ diameter [10] our model predicts that, ahead of a crack, cavitation would occur for maximum applied strain of 0.028 , associated with a global applied strain of around 0.014 (see Section 7.1). These results are, of course, approximate only since they have been deducted using identical material properties to the model rubber toughened epoxy. However, these results are useful in that they clearly predict cavitation of nano-sized 'rubber' particles. These ideas regarding the value of surface energy and the ease of cavitation of the rubber may be significant in the understanding of the toughness for epoxies toughened using multilayer core-shell particles [30].

\subsection{Energy Contributions}

Energy contributions from the different sources shown in equation 1 are shown in Figure 12. These results are for the cavitation of a $1.7 \mu \mathrm{m}$ radius particle as a function of strain. Figure 12a is for uniaxial loading and Figure 12b is for triaxial loading, plotted as a function of the maximum applied strain. The thermal energy is shown separately in these plots to show its small value, which is only significant for large particles which cavitate at low strains (see Section 7.6). For both types of loading, the elastic energy decreases after cavitation and the plastic energy increases, although this increase is 
very small for uniaxial loading and does not cause growth of shear bands as shown in Figure 10 . The surface energy is almost constant with increasing strain as the area inside the ellipsoid void, both for uniaxial and triaxial loading, hardly changes as it elongates although there is significant volume change, particularly for triaxial loading, as shown in Figure 8.

For uniaxial loading, particle cavitation occurs at 0.024 strain. From Figure 12a it is clear that the surface energy term dominates the energy balance. This observation shows agreement between our model and previous models which only included surface energy within the energy balance [e.g. 2]. Further, the hydrostatic stress in the rubber particle at cavitation is $22.3 \mathrm{MPa}$; this is very close to the hydrostatic stress value of $20 \mathrm{MPa}$ found to cause rubber particle cavitation using finite element modeling [9]. The energy contributions for triaxial loading are shown in Figure 12b; rubber particle cavitation is predicted to occur at 0.0116 maximum applied strain. Surface energy forms a significant term in the energy balance. The energy arising from the rubber particle is also significant for triaxial loading.

\subsection{Thermal Stress}

The thermal stress has been derived from the combination of the stresses arising from three elastic analyses using appropriate values of epoxy modulus for the temperature range. The values of coefficient of thermal expansion were not changed; for the epoxy, the value would be expected to approach the value used for the rubber for the higher temperature range. Neglecting temperature dependence in value of coefficient of thermal expansion may cause our calculations to be an overestimation of the total thermal stress. We have assumed that no thermal stress can be sustained above the glass transition temperature of the epoxy matrix. This seems a reasonable assumption taking into account the ability of the materials to relax at high temperatures.

It is notable that our calculations lead to modest and highly concentrated thermal stress in the epoxy (see Figure 5); however, the rubber particle is placed in significant hydrostatic tension of 9.17 MPa. It is notable that these thermal stresses are far smaller than those which would be found using a single elastic analysis for the entire temperature drop using room temperature properties.

The effect of thermal stress on the cavitation criterion for uniaxial and triaxial cavitation is shown in Figure 16. For both types of loading, the effect is significant for larger particles when the stresses are low and the thermal energy contribution is therefore significant. The results in Figure 16 show that the 
effect may be significant for epoxies toughened with conventional sized particles. For the epoxy toughened with rubber particles of $1.7 \mu \mathrm{m}$, cavitation in triaxial loading, including thermal stress, is predicted at 0.0116 maximum applied strain; without thermal stress, cavitation is predicted at 0.0144 maximum applied strain. This approximately $25 \%$ increase in strain may be significant for some applications when optimised toughness is not obtained by, for example, constraint along a relatively thin adhesive layer in a bonded joint. In such conditions, cavitation and thus optimised toughness for such adhesives may be reliant on thermal stress. These observations regarding the important role of thermal stress may be significant for the long-term properties of such adhesives. Thermal stress in polymers is expected to relax over prolonged time periods. The long-term toughness of adhesive joints manufactured using toughened epoxies with conventional sized rubber particles is therefore worthy of investigation.

\subsection{Strain Distributions}

The increase in shear strain following cavitation of the rubber particle in triaxial loading has been shown in Figure 9; these results are from cavitation at 0.0116 maximum applied strain, as predicted for the $1.7 \mu \mathrm{m}$ radius particle. At higher applied strains, more complex shear band growth is found as shown in Figure 17; two distinct shear bands between neighbouring particles are found. Such particleparticle interaction behaviour is found for a maximum applied strain of 0.0314 , but not for 0.0234 strain. Using the results in Figure 13, such double shear bands would be expected for cavitation of particles with radius less than about $140 \mathrm{~nm}$. This extensive deformation would be expected to contribute to higher plastic energy and thus increasing toughness. These observations may indicate a mechanism of increased toughness as materials are optimised.

\section{Concluding Remarks}

Cavitation of rubber particles in rubber toughened epoxies is an important process in developing the toughness of these materials. A criterion for particle cavitation has been derived which is based on experimental observations and finite element simulations of the cavitation process. This criterion leads to the prediction of a particle size effect. Cavitation of rubber particles ahead of a crack occurs at a lower global applied stress compared with uniaxial loading. The effect of thermal stresses may be significant for epoxies toughened using conventional sized particles. The criterion has been extended for nano-sized 'rubber' particles using a different value of surface energy for the void. The derivation of a criterion for rubber particle cavitation may be of crucial importance in present developments of advanced materials containing varying sized particles. 


\section{Acknowledgements}

This project was jointly funded by the Engineering and Physical Sciences Research Council, UK, and the Defence Science and Technology Laboratory, UK. We gratefully acknowledge the contributions to the Finite Element simulations from Dr Xiaowei Wang and Dr Nadia Balhi, University of Bristol, UK. We thank Dr Bernt Johnsen and Spyros Spyridoulias, Imperial College London, UK, and Dr G.D. Dean, National Physical Laboratory, UK, for providing experimental results. We thank Dr D.L. Hunston, National Institute of Standards and Technology, USA, and Professor J.G. Williams, Imperial College London, UK, for valuable discussions. 


\section{References}

1. Kinloch AJ. MRS Bulletin 2003;28:445-448

2. Lazzeri A, Bucknall CB. Polymer 1995;36:2895-2902

3. Fond C. J. Polymer Physics 2001;39:2081-2096

4. Dompas D, Groeninckx G, Isogawa M, Hasegawa T, Kadokura M. Polymer 1994;35:47504759

5 Yee AF, Pearson RA. J. Mater. Sci. 1986;21:2462-2474

6. Pearson RA, Yee AF. J. Mater. Sci. 1986;21:2475-2488

7. Pearson RA, Yee AF. J. Mater. Sci. 1991;26:3828-3844

8. Bagheri R, Marouf BT, Pearson RA. Polymer Reviews 2009;49:201-225

9. Gehant S, Fond C, Schirrer R. Int. J. Fracture 2003;122:161-175

10. Liu J, Sue H-J, Thompson ZJ, Bates FS, Dettloff M, Jacob G, Verghese N, Pham H. Macromolecules 2008;41:7616-7624

11. Kunz SC, Beaumont PWR. J. Mater. Sci. 1981;16:3141-3152

12. Hibbitt, Karlsson and Sorensen. ABAQUS/Standard user manual 2002: version 6.3

13. Yasmin A, Daniel IM. Polymer 2004;45:8211-8219

14. Mamlouk MS, Witczak MW, Kaloush K E, Hasan N. J. Testing and Evaluation 2005;33:118126

15. Oba T. PhD thesis 1999: Imperial College London

16. Kinloch AJ, Shaw SJ, Tod DA, Hunston DL. Polymer 1983;24:1341-1354

17. Duncan B, Dean GD. Int. J. Adhesion and Adhesives 2003;23:141-149

18. Huang Y, Kinloch AJ. J. Mater. Sci. Letters 1992;11:484-487

19. Guild FJ, Kinloch AJ. J. Mater. Sci. 1995;30:1289-1697

20. Guild FJ, Kinloch AJ, Taylor AC. 'A Predictive Model for the toughness of rubber toughened epoxies’; paper in preparation

21. Levy N, Marcel PV, Ostergren WJ, Rice JR. J. Fracture Mech. 1971;7:143-156

22. Rice JR. J. Appl. Mech. 1968;35:379-386

23. Davy PJ, Guild FJ. Proc. Roy. Soc. A 1988;418:95-112

24. Bucknall CB, Karpodinis A, Zhang XC. J. Mater. Sci. 1994;29:3377-3383

25. Lake GJ, Thomas AG. Proc. Roy. Soc. A 1967;300:108-119

26. Almer CJ, Pocius AV. Proc. SAMPE Technical Conf. Seattle, USA 1980

27. Gent AN. Rubber Chemistry and Technology 1990;63:49-53

28. Smit RJM, Brekelmans WAM, Meijer HEH. J. Mater. Sci. 2000;35:2869-2879 
29. Kim DS, Cho K, Kim JK, Park CE. Polymer Engin. and Sci. 1996;36:755-768

30. Day RJ, Lovell PA, Wazzan AA. Polymer Int. 2001;50:849-857 


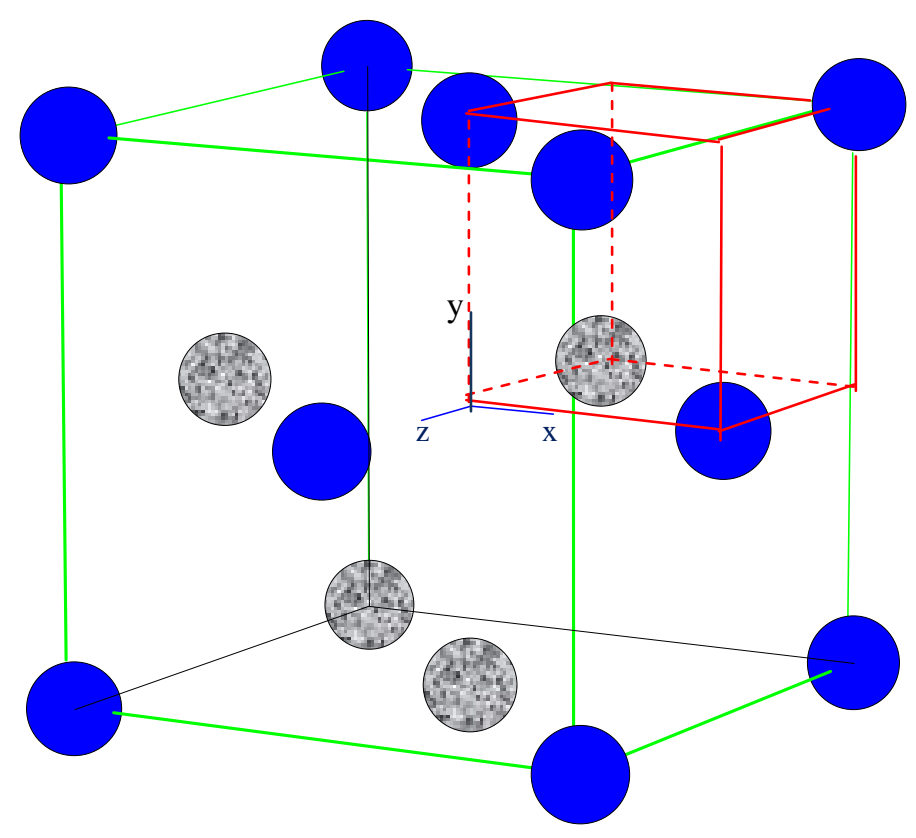

Figure 1a 


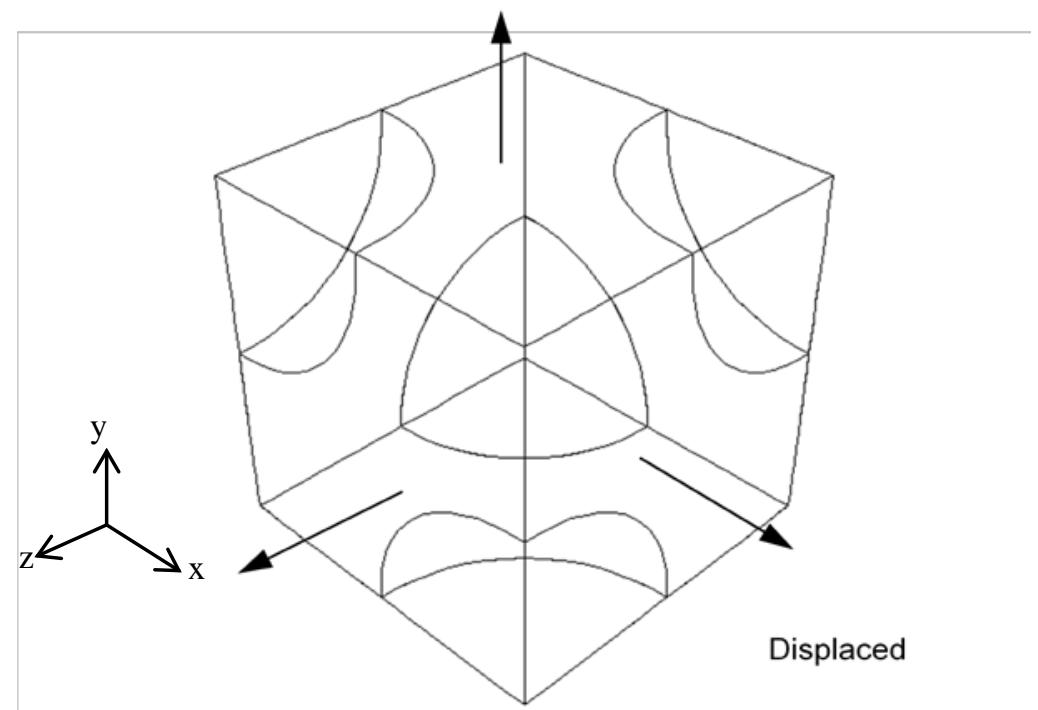

Figure 1b 


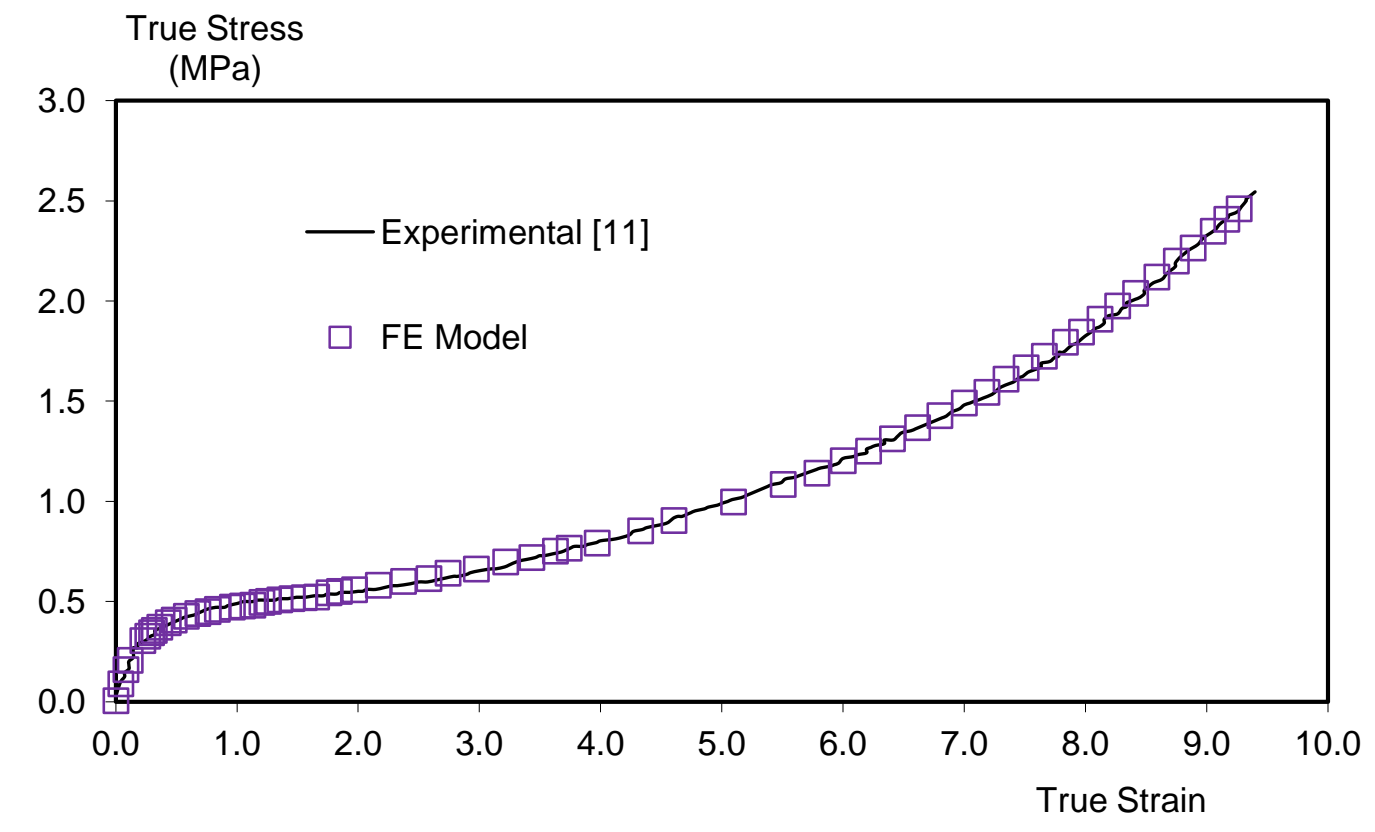

Figure 2a 


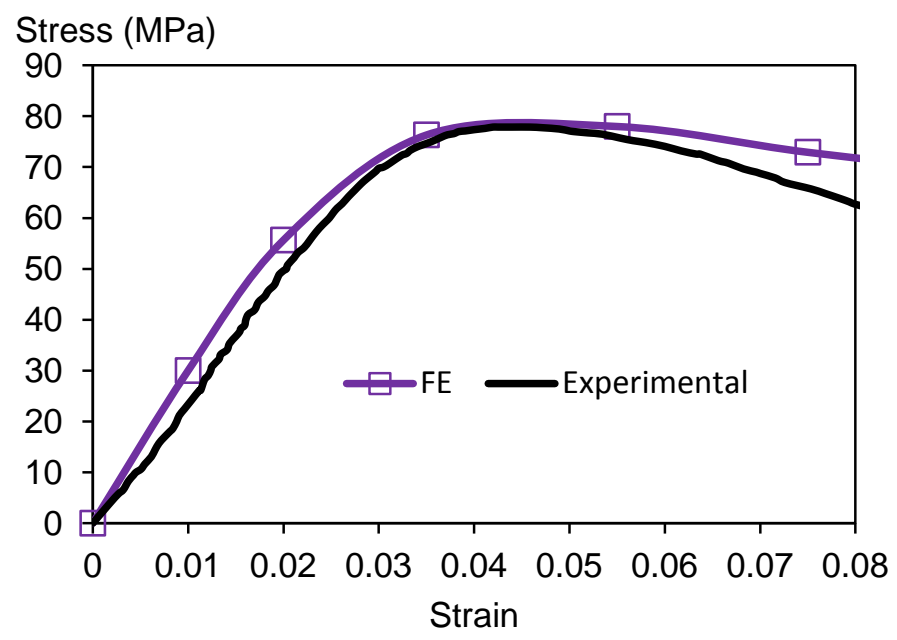

Figure 2b 


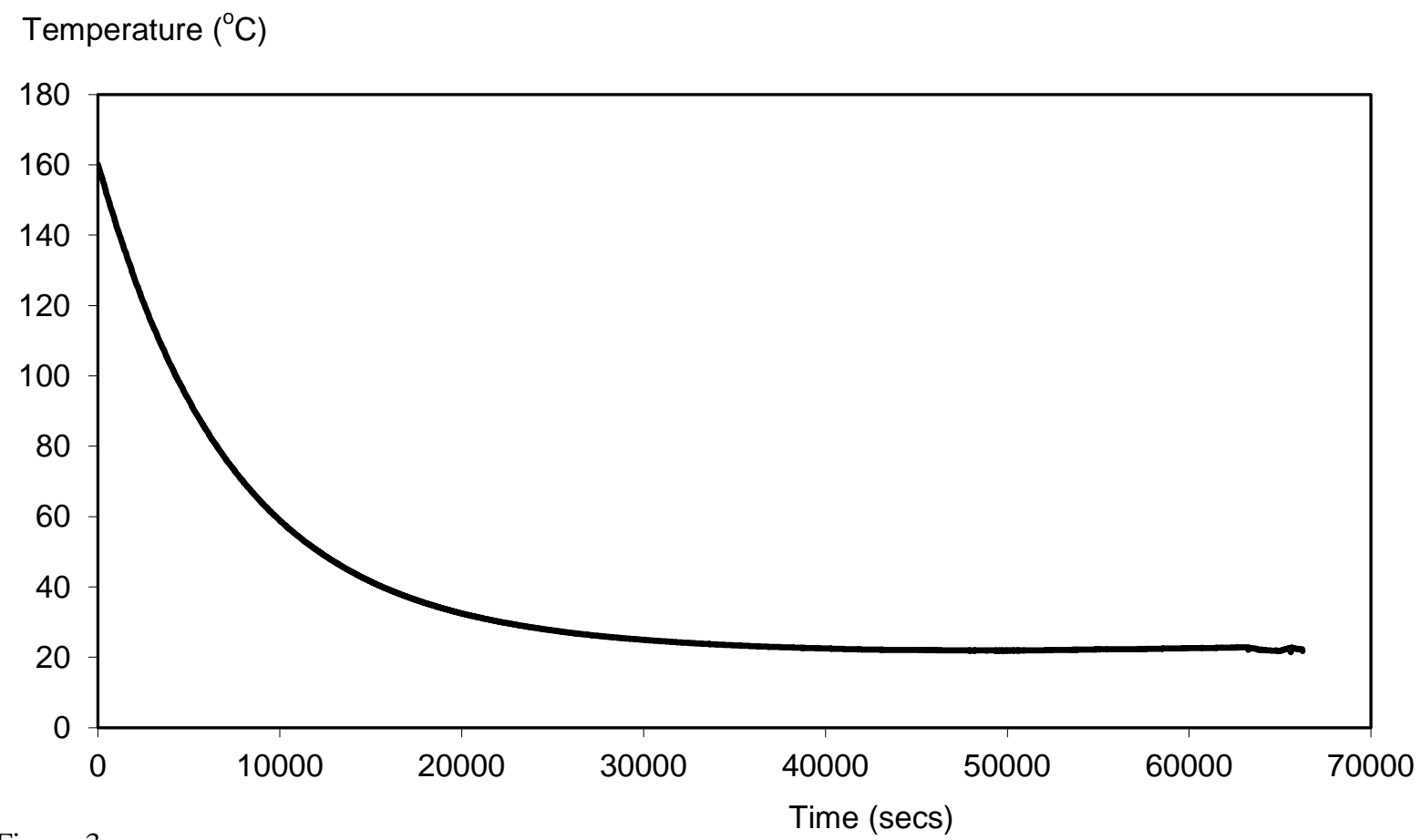

Figure 3a 


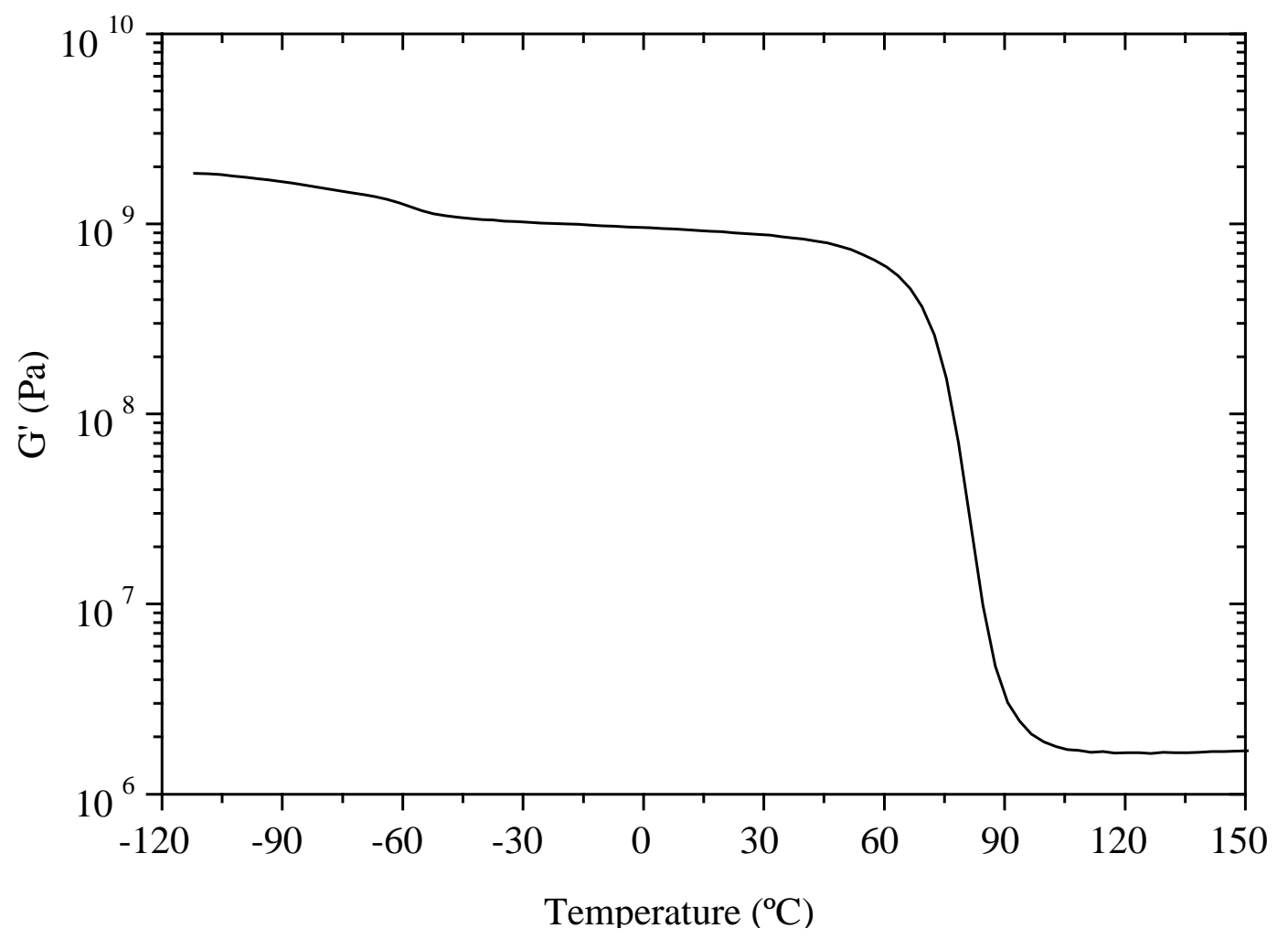

Figure 3b 
Temperature $\left({ }^{\circ} \mathrm{C}\right)$

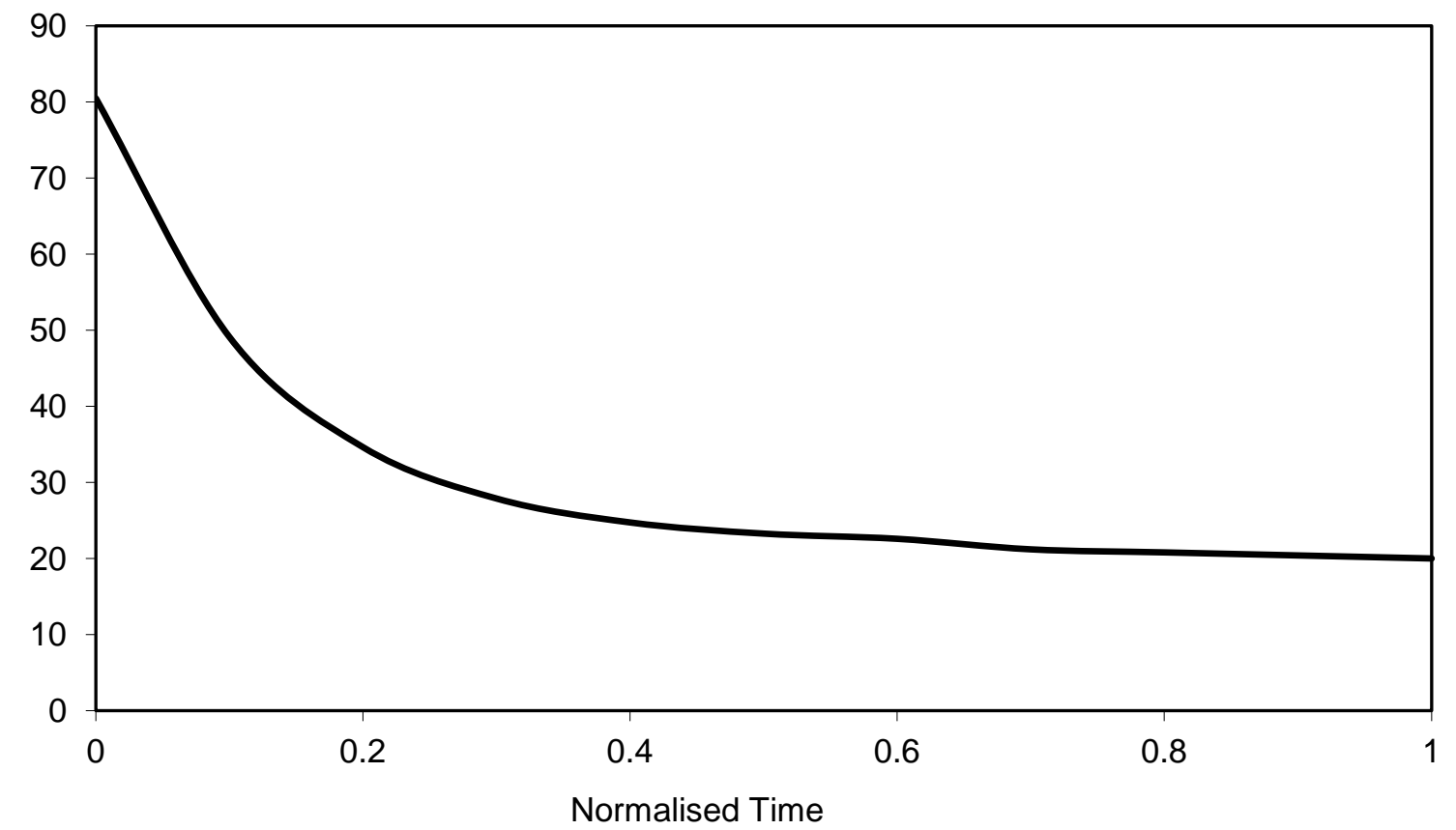

Figure 4a 


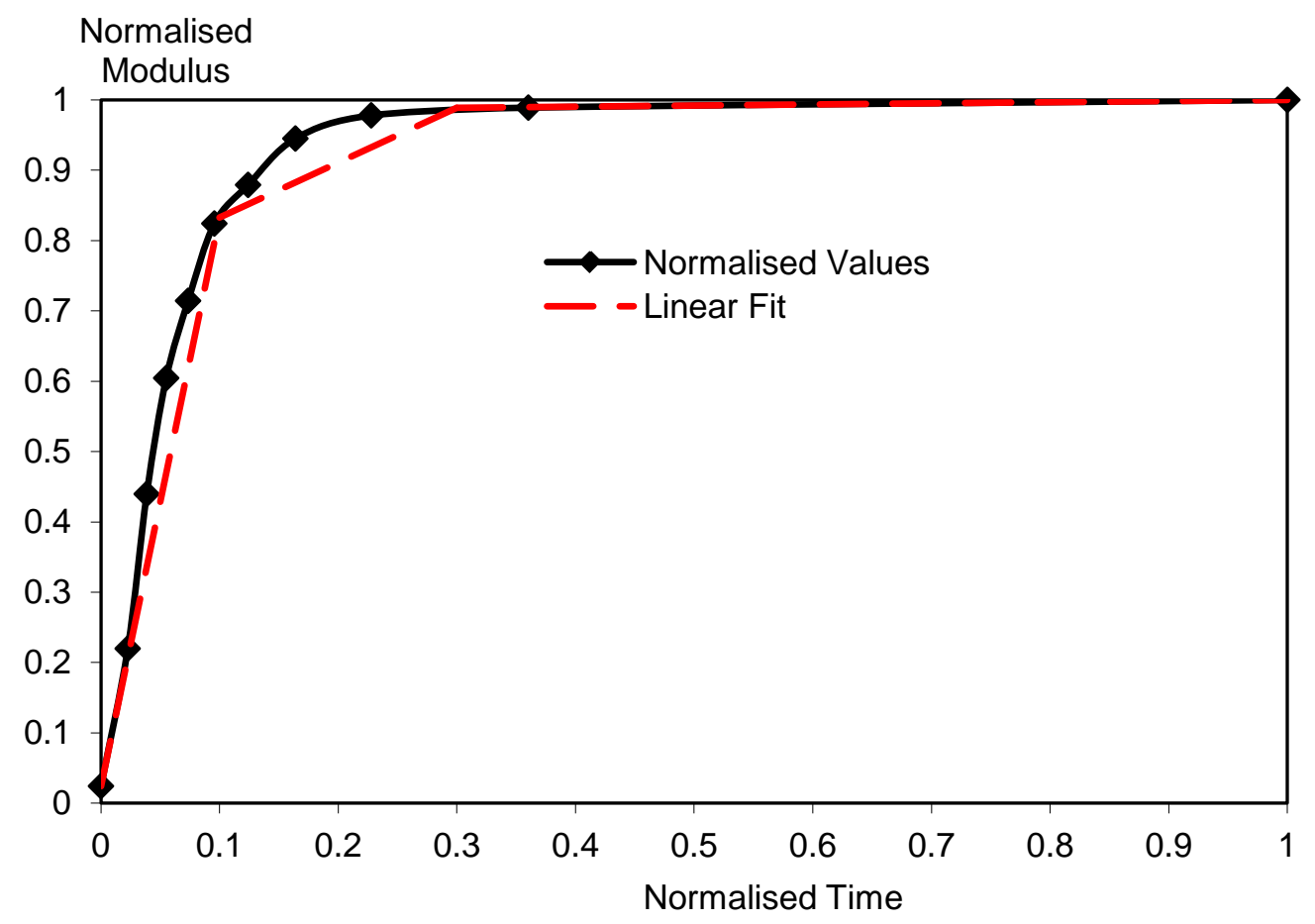

Figure 4b 


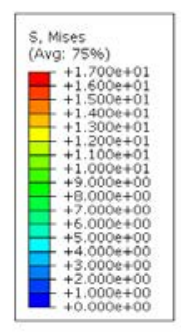

ב文

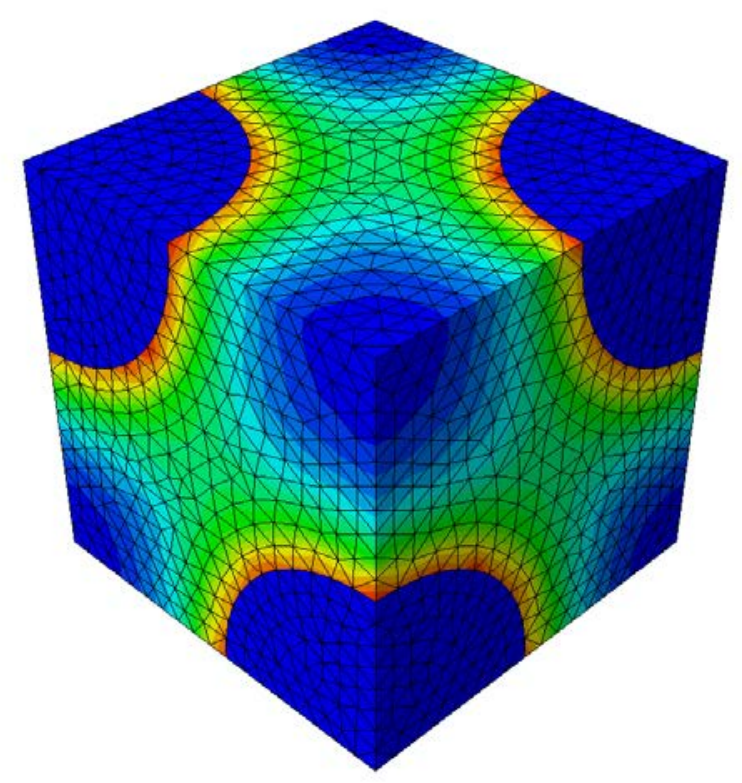

Figure 5 
Stress (MPa)

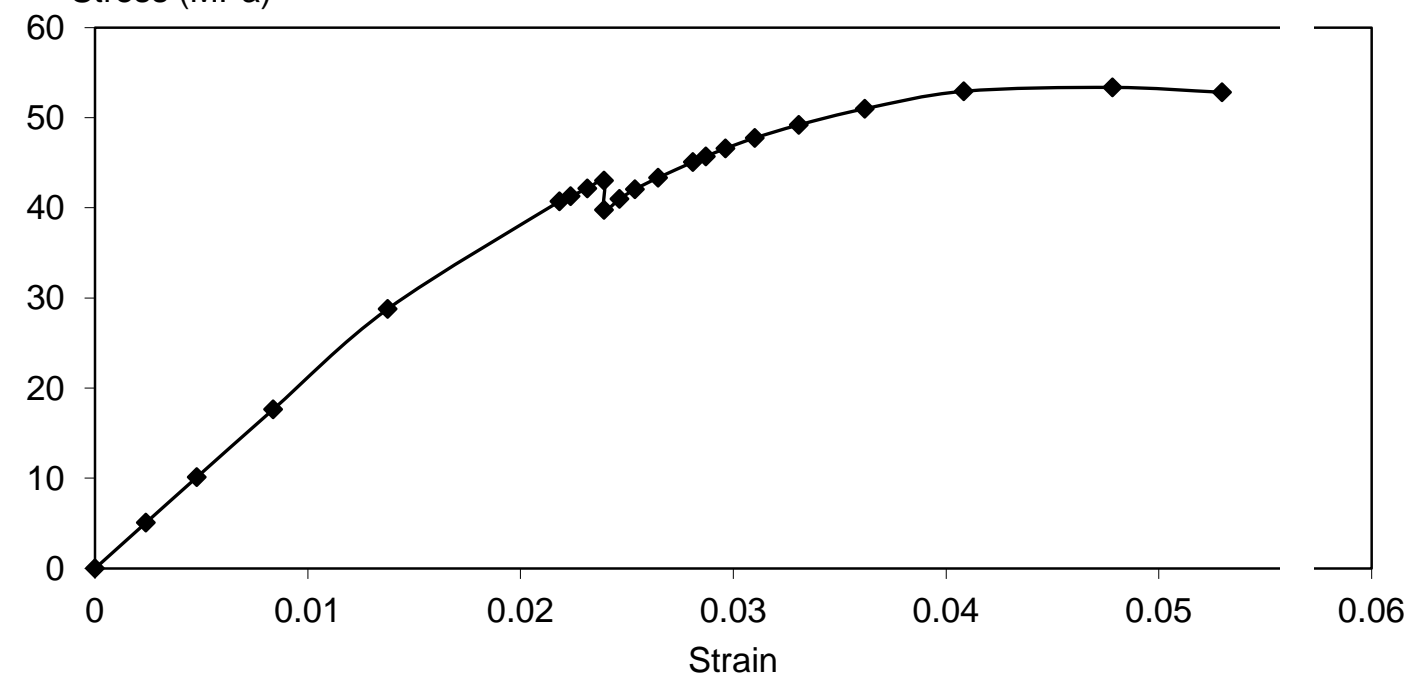

Figure 6a 


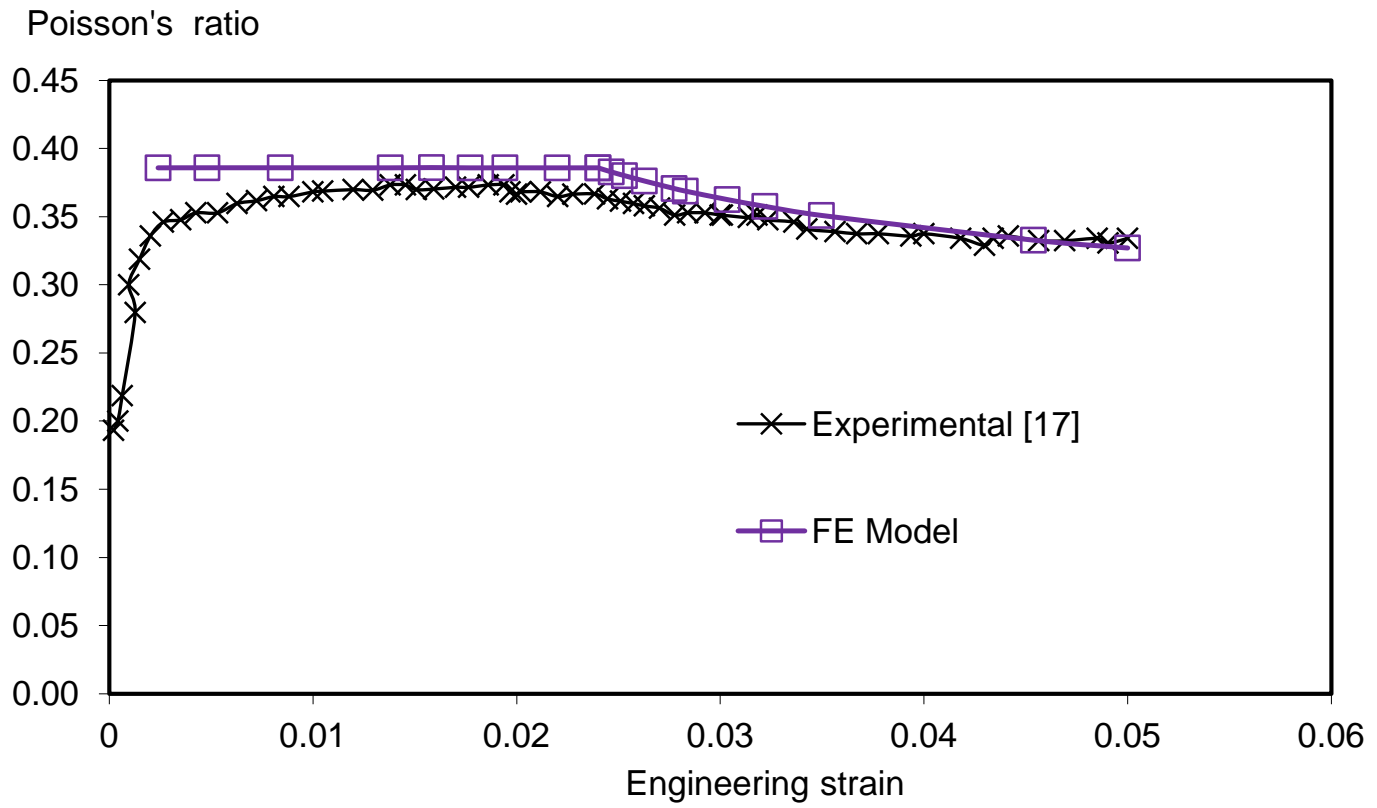

Figure 6b 




Figure 7a 


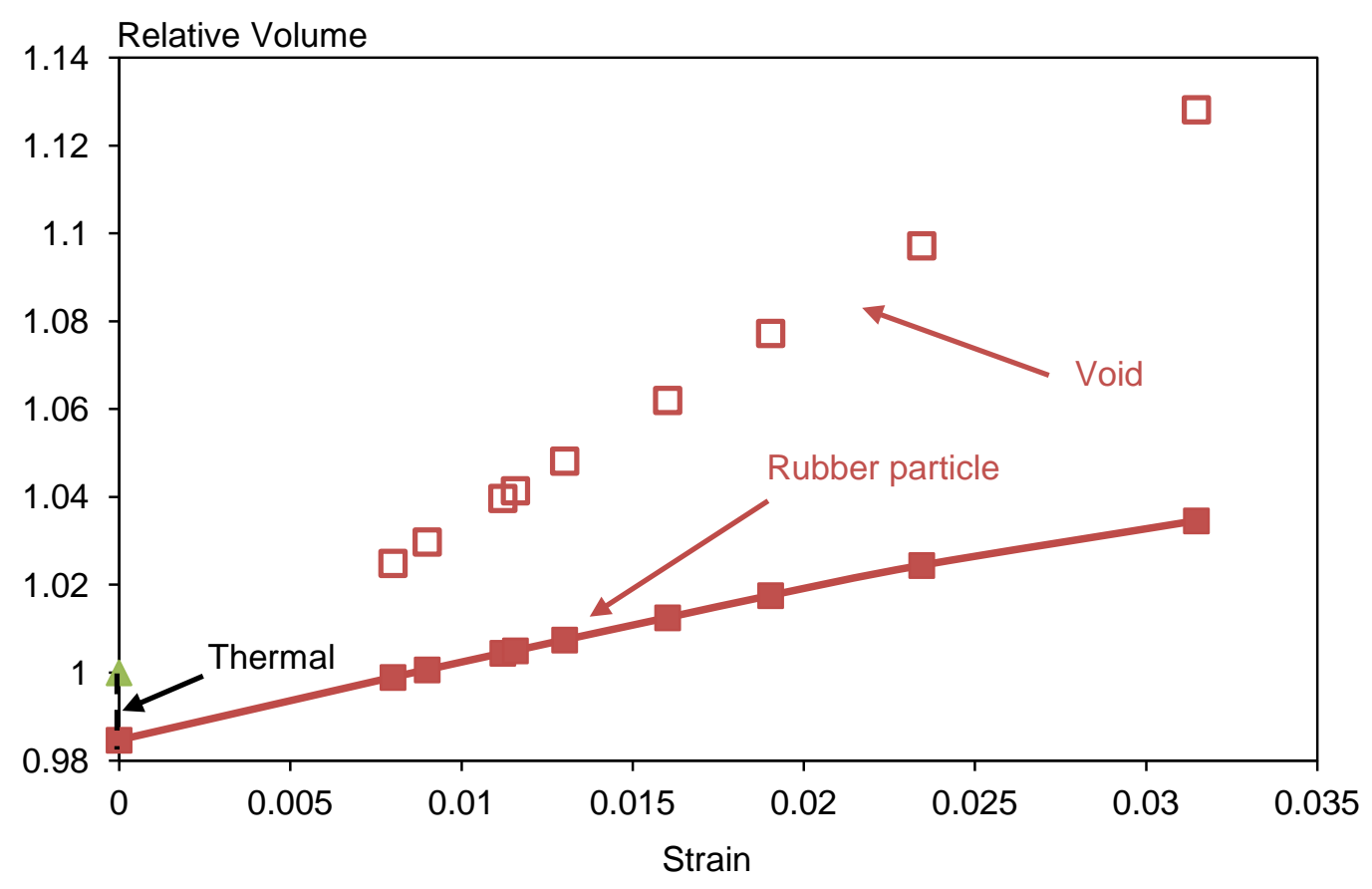

Figure 7b 


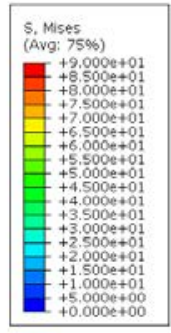

之文

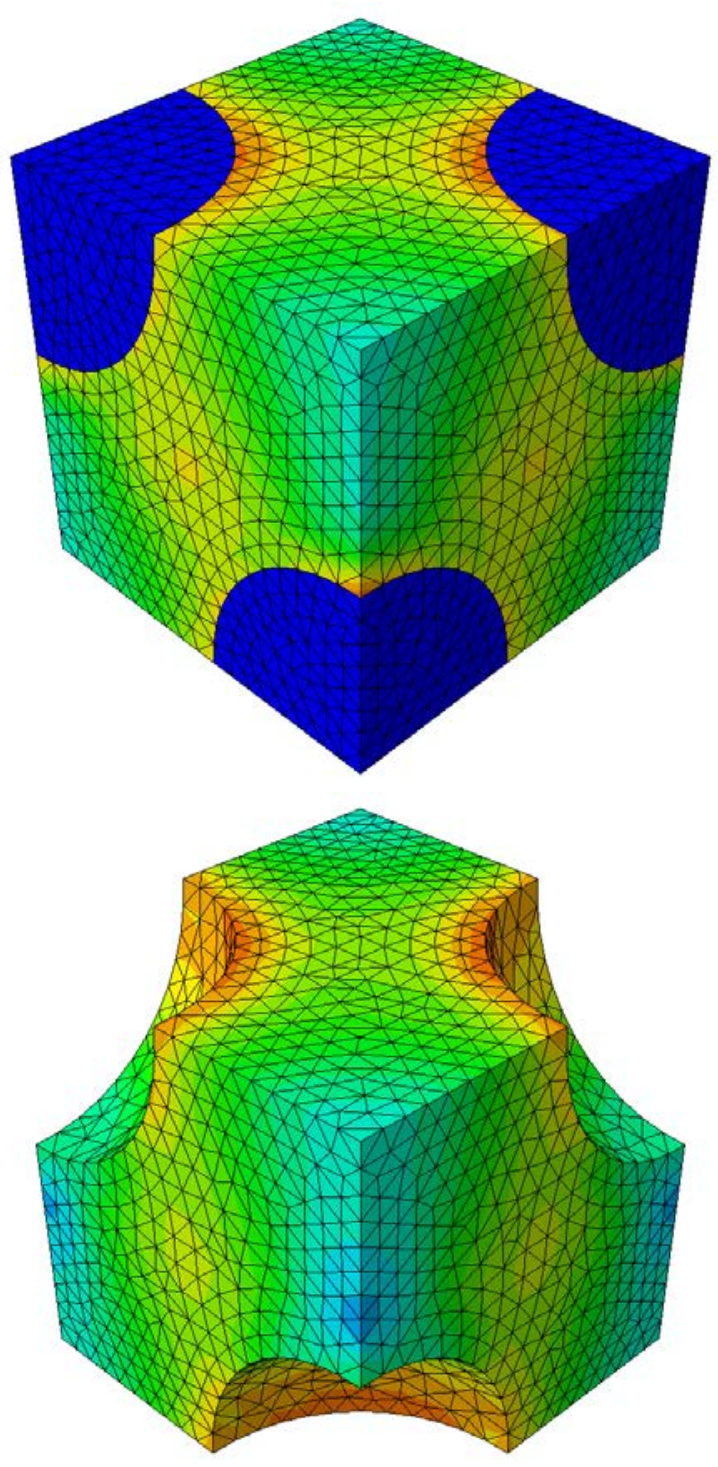

Figure 8 


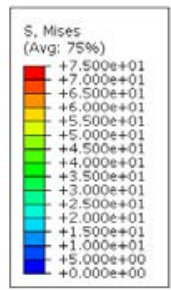

之只
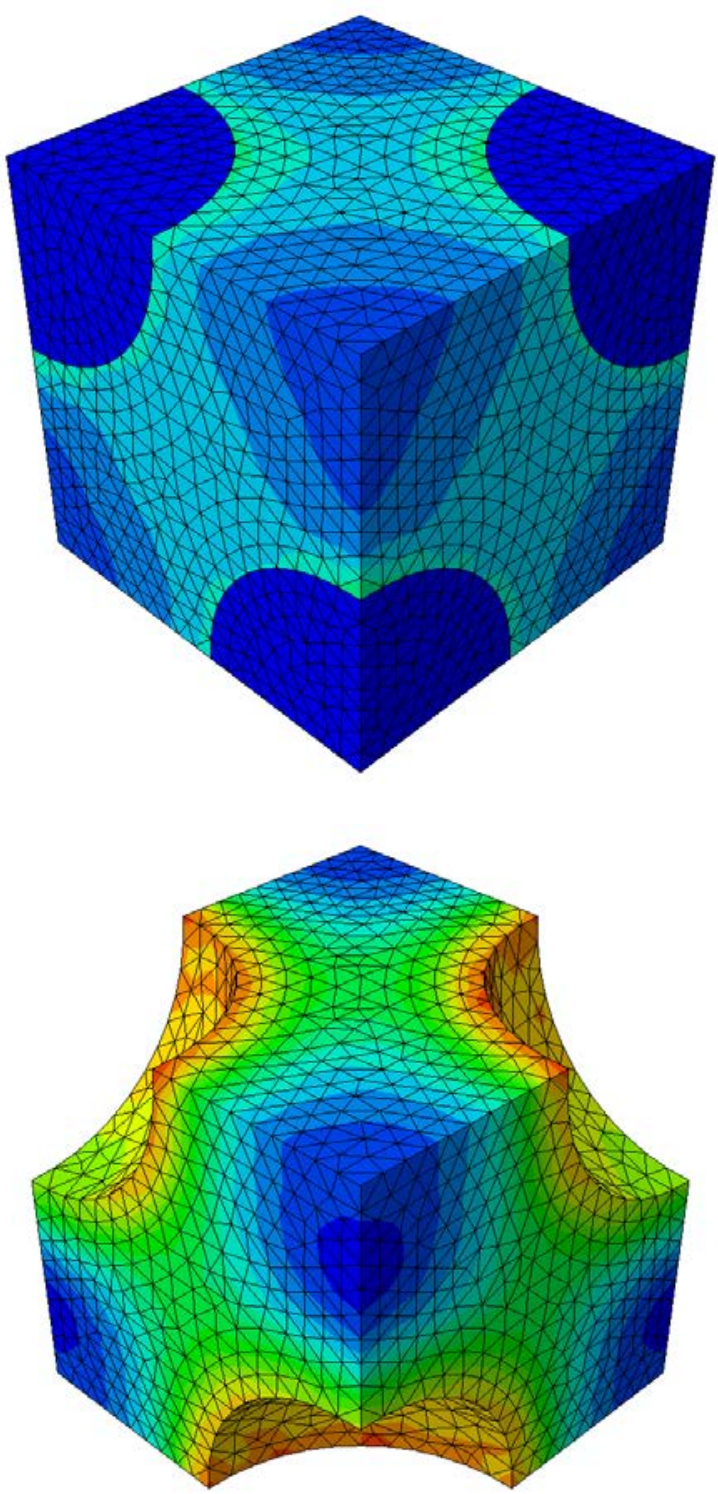

Figure 9 


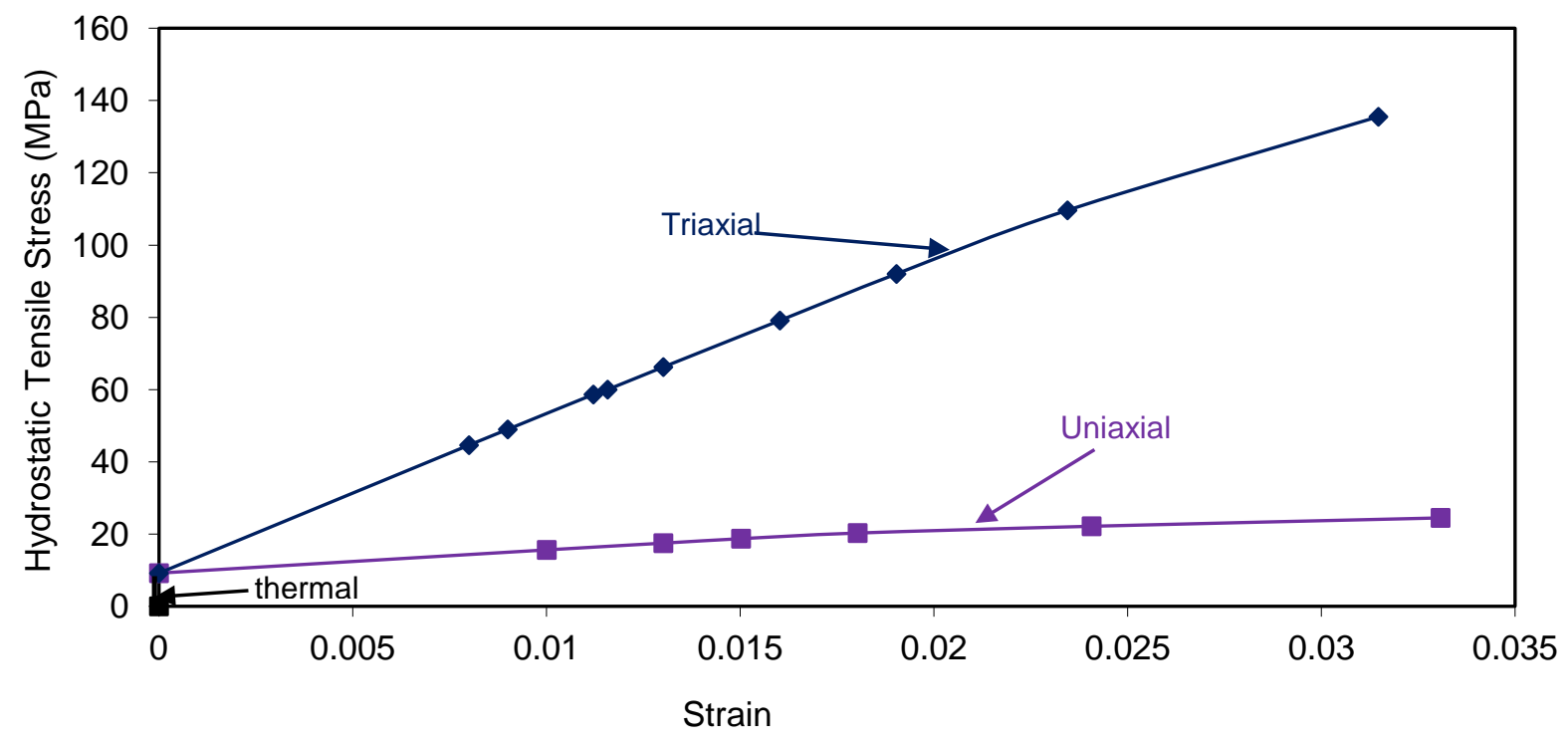

Figure

10 


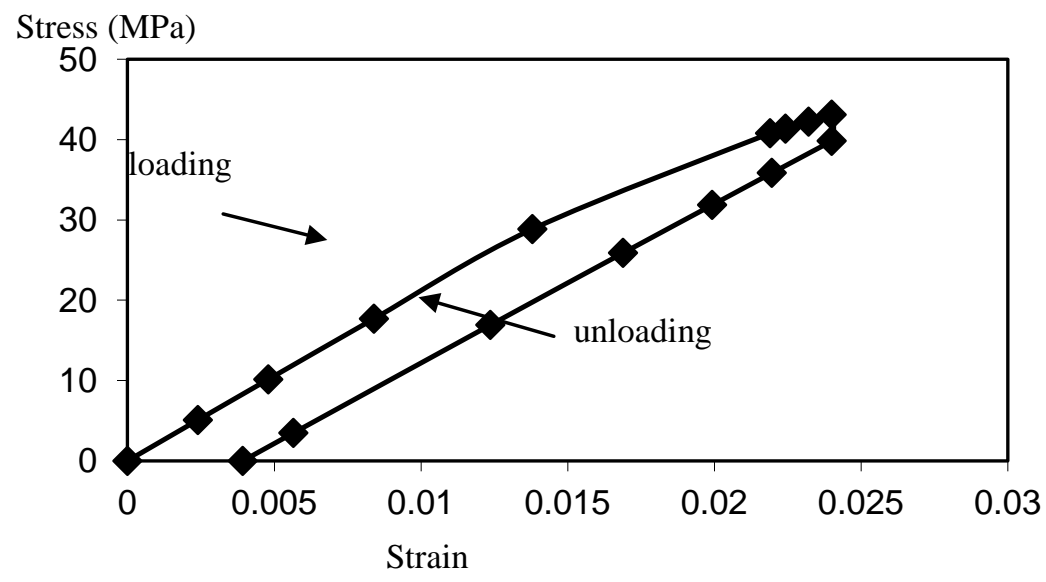

Figure 11 


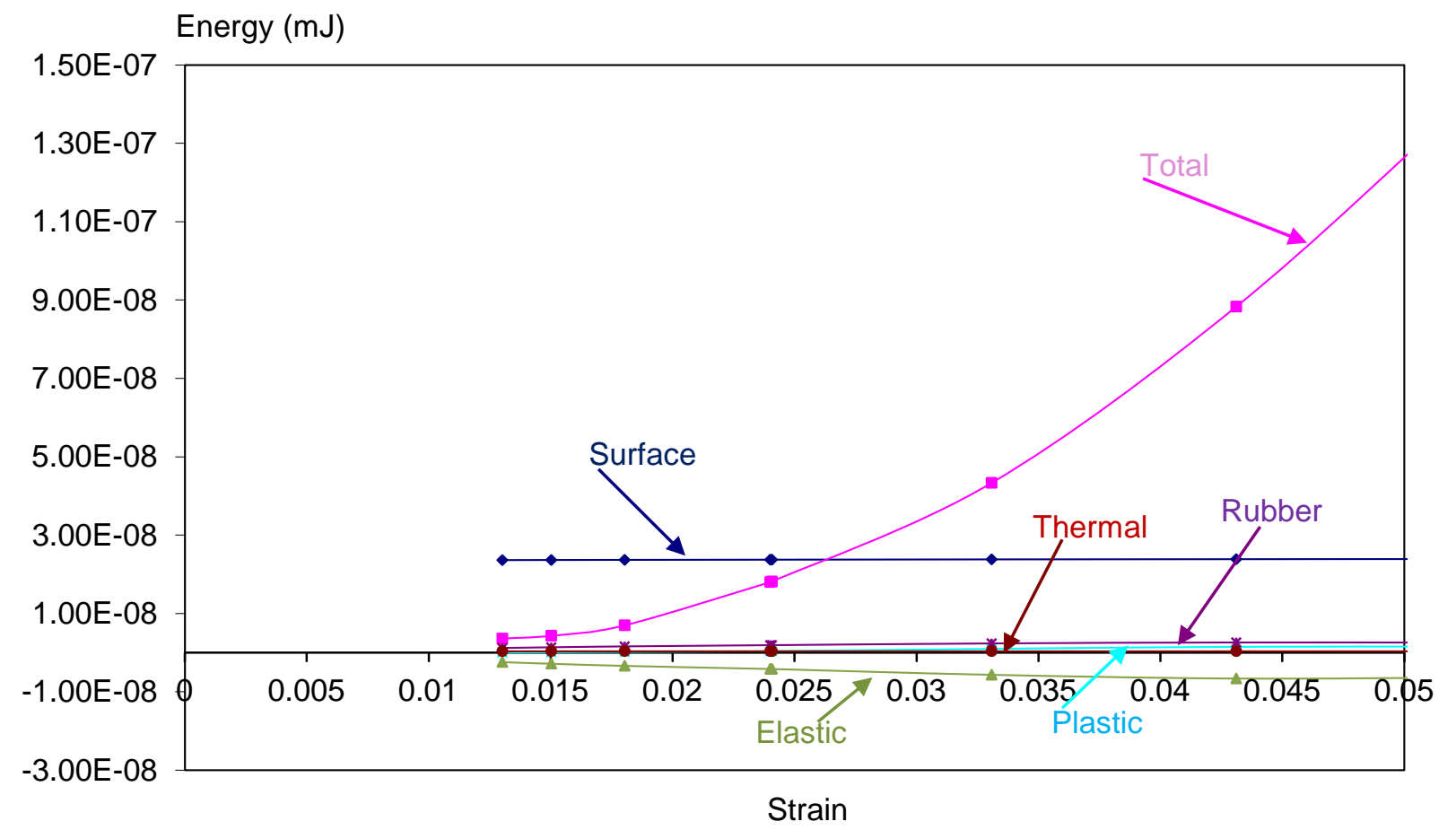

Figure 12a 


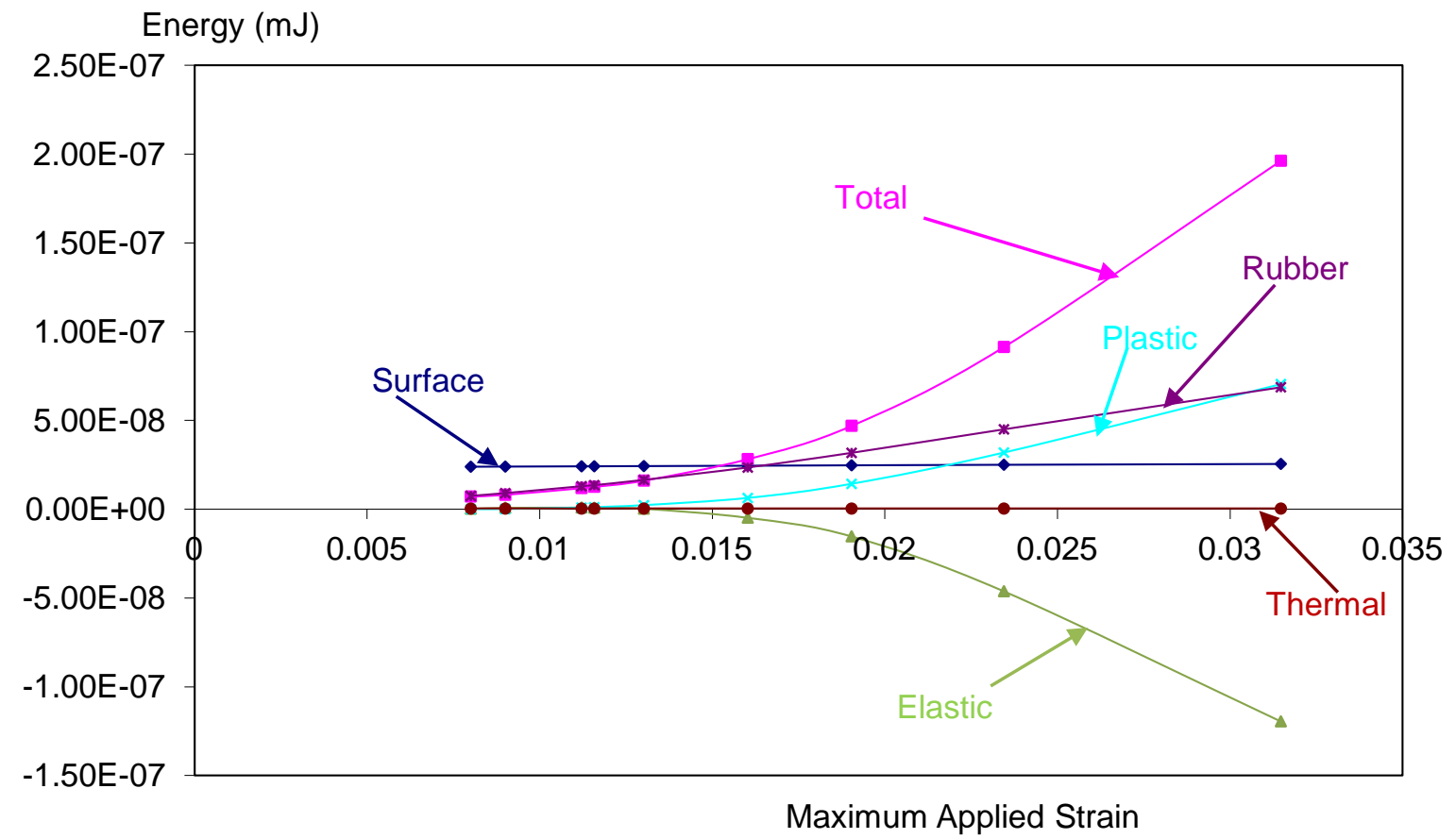

Figure 12b 




Figure 13 


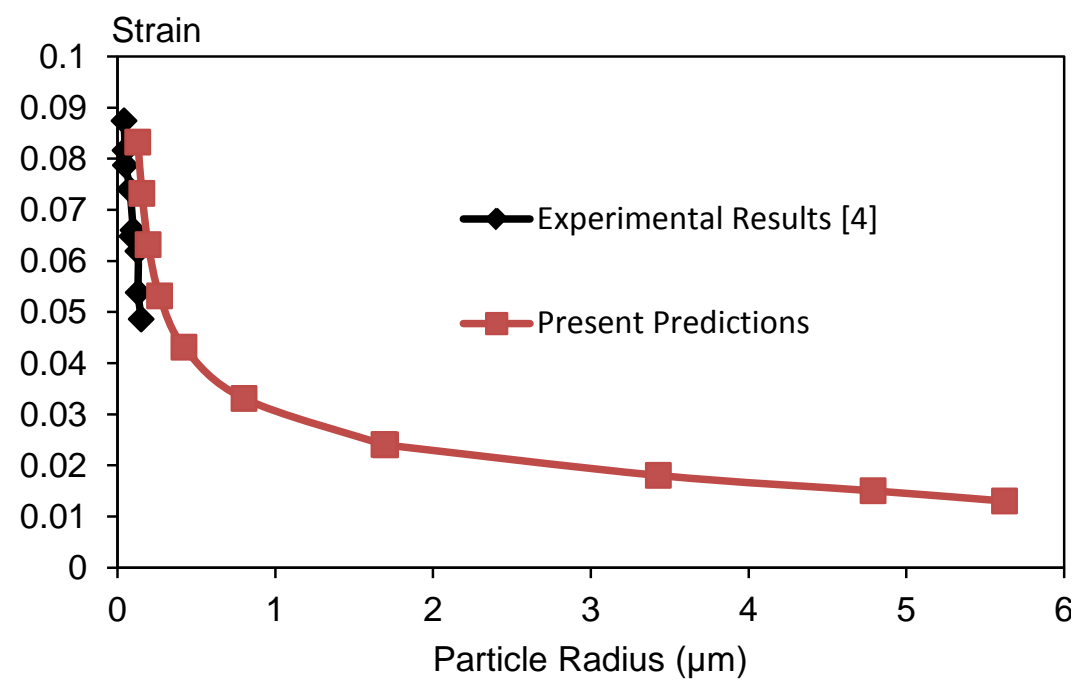

Figure 14 


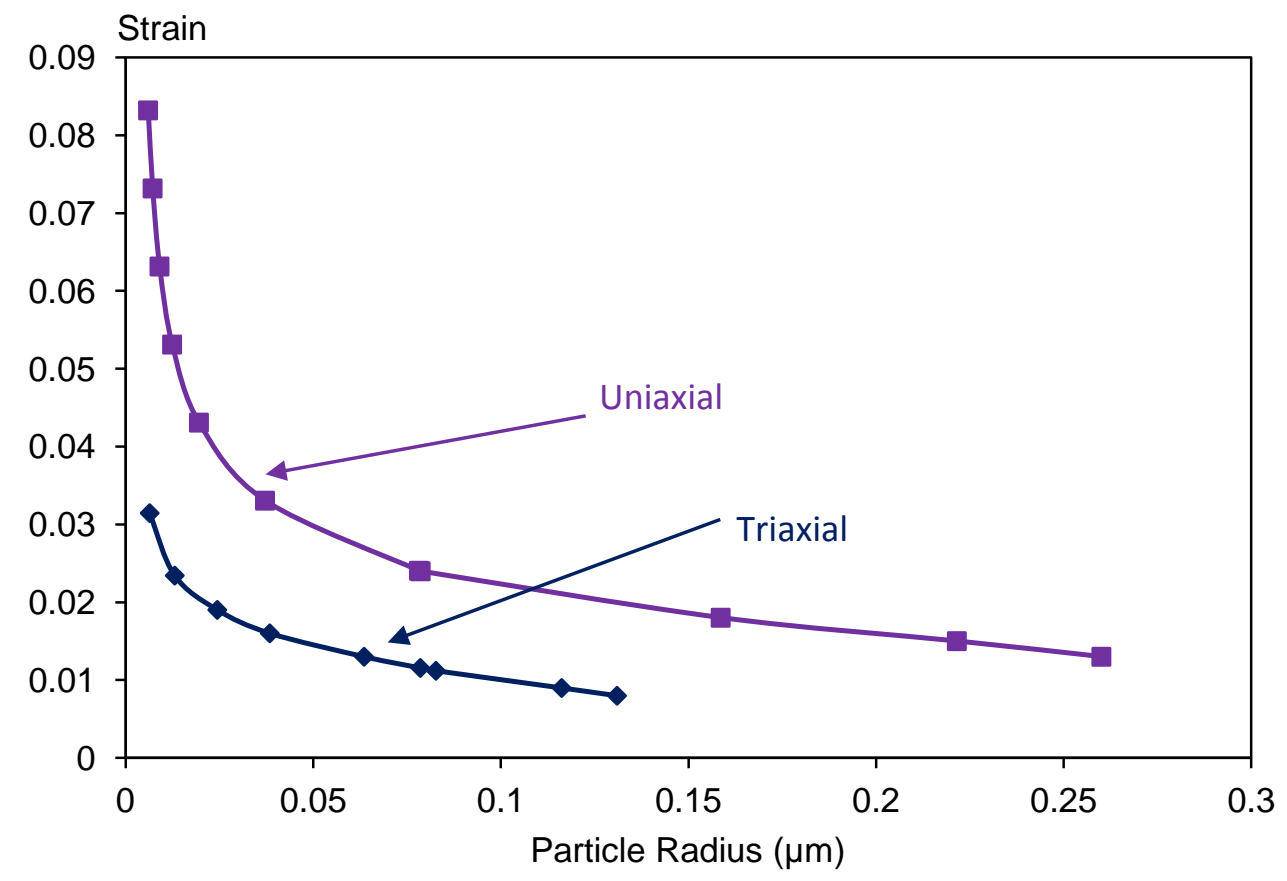

Figure 15 


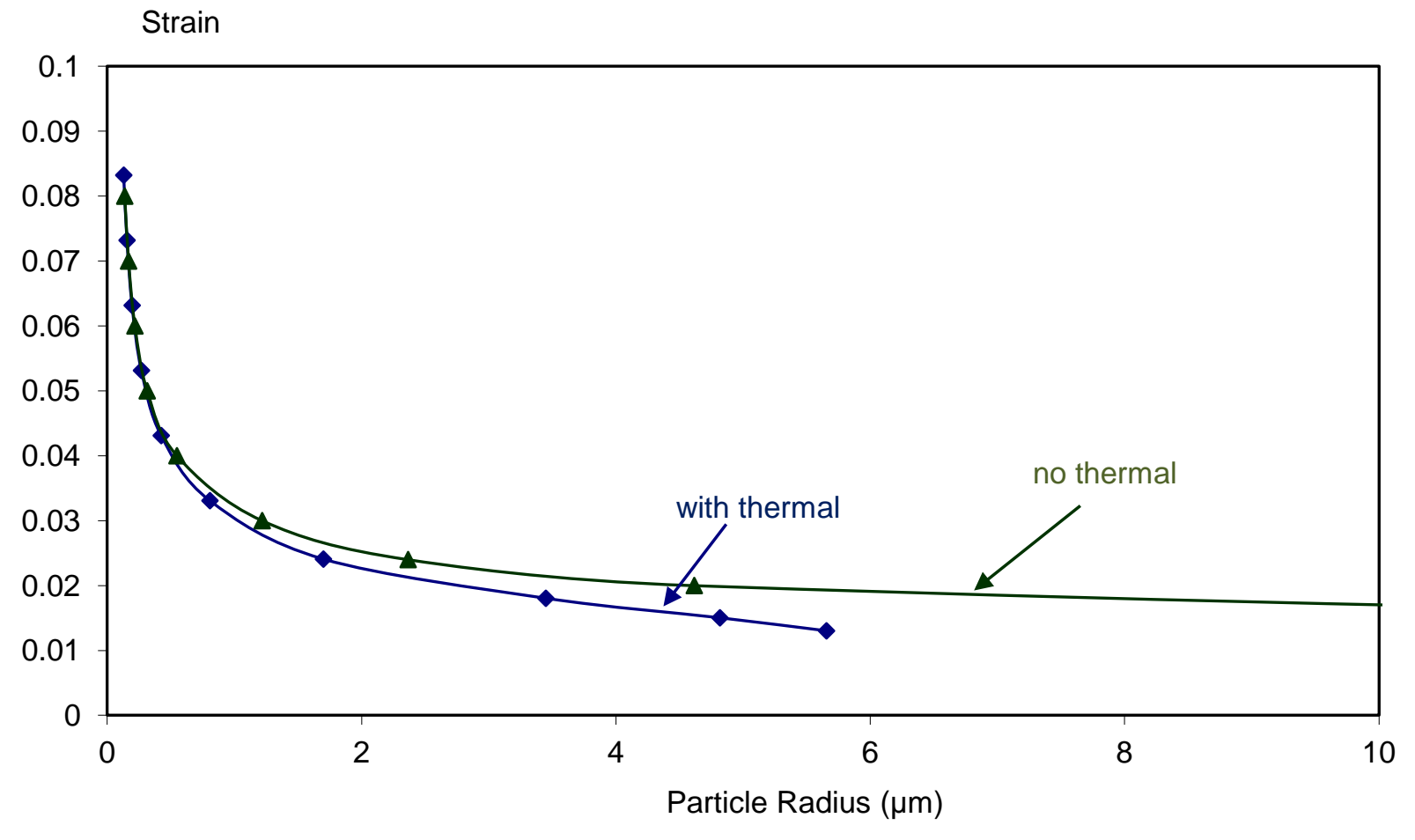

$16 a$

Figure 


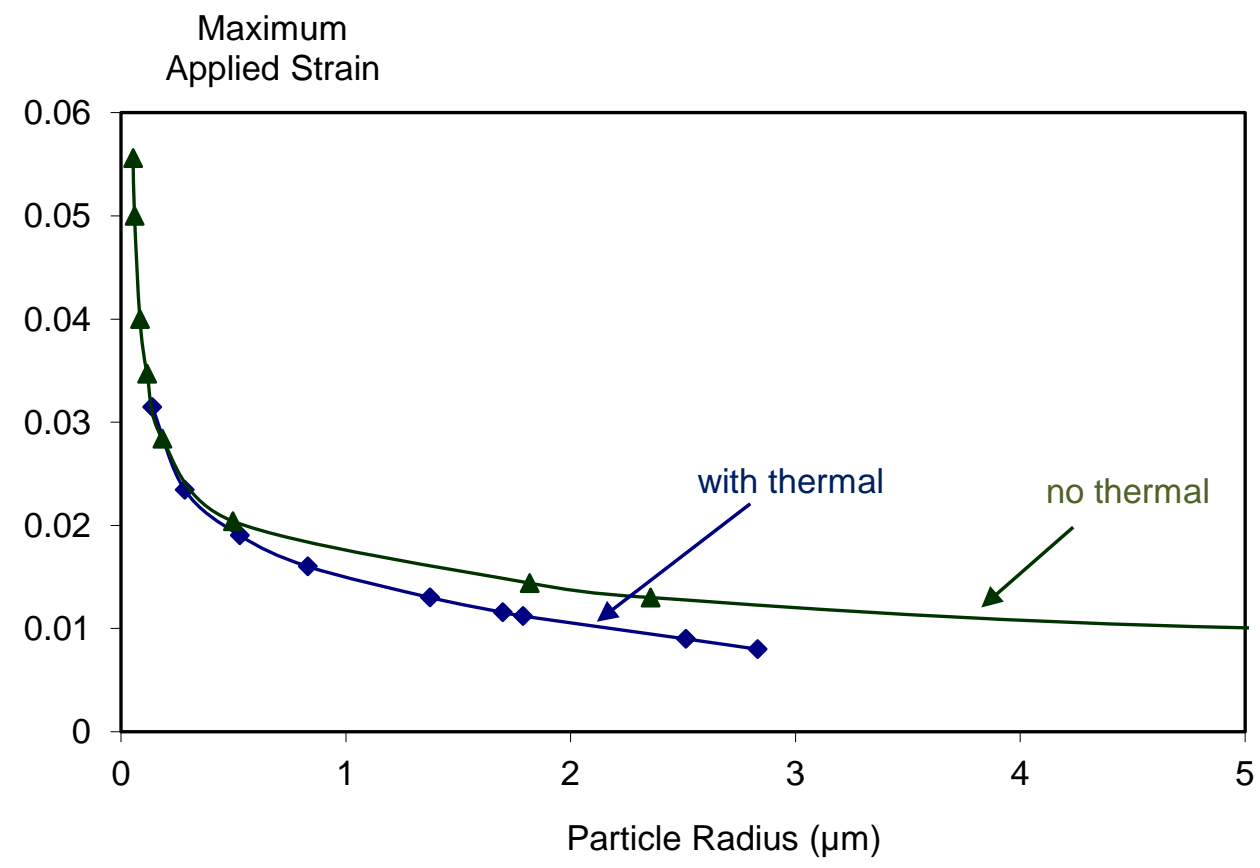

Figure 16b 

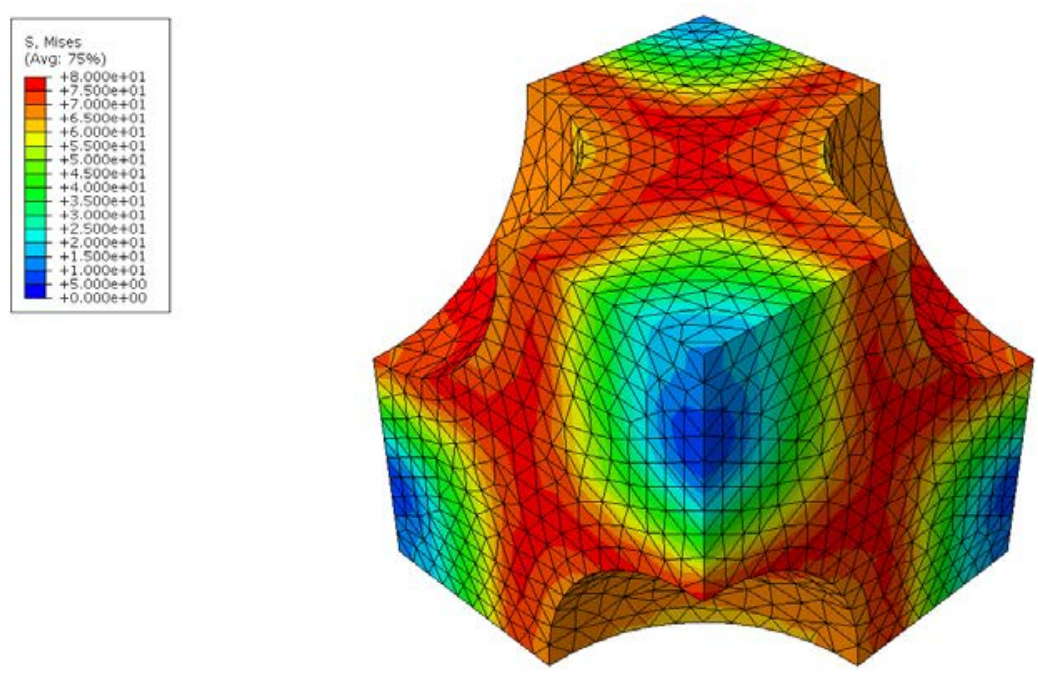

$\gamma_{x}$

Figure 17 


\section{$\underline{\text { List of Figures }}$}

Figure 1 Development of the Unit Cell

Figure 2 Comparison of Experimental and Modelled Values of Material Properties

a) Tensile stress/strain of rubber [11]

b) Tensile stress/strain of epoxy

Figure 3 Cooling during cure

a) Variation of temperature during cooling

b) Shear Modulus variation with temperature measured using DMTA [15]

Figure 4 Normalisation of cooling data

a) Variation of temperature

b) Variation of modulus

Figure 5 Contours of von Mises stress arising from thermal strains (stress range: $0-17 \mathrm{MPa}$ )

Figure 6 Mechanical properties for Uniaxial cavitation at 0.024 applied strain

a) Predicted stress/strain behaviour

b) Comparison of Predicted and Measured [17] values of Poisson's Ratio

Figure 7 Comparison of Particle and Void Volume after cavitation at increasing applied strain

a) Uniaxial loading

b) Triaxial loading

Figure 8 Contours of von Mises stress for Uniaxial Cavitation at 0.024 applied strain (stress range: 0$90 \mathrm{MPa})$

a) Before cavitation

b) After cavitation

Figure 9 Contours of von Mises stress for Triaxial Cavitation at 0.0116 maximum applied strain (stress range: 0-75 $\mathrm{MPa}$ )

a) Before cavitation

b) After cavitation

Figure 10 Variation of Hydrostatic Stress in Rubber Particle for Uniaxial and Triaxial Loading

Figure 11 Predicted Stress/strain behaviour for Uniaxial Cavitation at 0.024 applied strain followed by unloading.

Figure 12 Variation of Energy Contributions from Cavitation of $1.7 \mu$ m radius particle with applied strain

a) Cavitation under uniaxial strain

b) Cavitation under triaxial strain 
Figure 13 Cavitation Criterion showing effect of particle size for Uniaxial and Triaxial Loading

Figure 14 Uniaxial Cavitation Criterion; comparison with results from Dompas et al [4]

Figure 15 Preliminary Caviation Criterion showing effect of block co-polymer nano-sized particles for Uniaxial and Triaxial Loading (surface energy of void $=0.03 \mathrm{~J} \mathrm{~m}^{-2}$ )

Figure 16 Effect of thermal stress on Cavitation Criterion
a) Uniaxial Loading
b) Triaxial Loading

Figure 17 Contours of von Mises stress after rubber particle cavitation at 0.0314 strain (stress range: 0-80 MPa) 